University of Nebraska - Lincoln

DigitalCommons@University of Nebraska - Lincoln

\title{
DLVO Interaction Energies between Hollow Spherical Particles and Collector Surfaces
}

\author{
Chongyang Shen \\ China Agricultural University \\ Scott Bradford \\ USDA-ARS, Scott.Bradford@ars.usda.gov \\ Zhan Wang \\ Shenyang Agricultural University \\ Yuanfang Huang \\ China Agricultural University \\ Yulong Zhang \\ Shenyang Agricultural University \\ See next page for additional authors
}

Follow this and additional works at: https://digitalcommons.unl.edu/usdaarsfacpub

Shen, Chongyang; Bradford, Scott; Wang, Zhan; Huang, Yuanfang; Zhang, Yulong; and Li, Baoguo, "DLVO Interaction Energies between Hollow Spherical Particles and Collector Surfaces" (2017). Publications from USDA-ARS / UNL Faculty. 1791.

https://digitalcommons.unl.edu/usdaarsfacpub/1791

This Article is brought to you for free and open access by the U.S. Department of Agriculture: Agricultural Research Service, Lincoln, Nebraska at DigitalCommons@University of Nebraska - Lincoln. It has been accepted for inclusion in Publications from USDA-ARS / UNL Faculty by an authorized administrator of DigitalCommons@University of Nebraska - Lincoln. 


\section{Authors}

Chongyang Shen, Scott Bradford, Zhan Wang, Yuanfang Huang, Yulong Zhang, and Baoguo Li 


\title{
DLVO Interaction Energies between Hollow Spherical Particles and Collector Surfaces
}

\author{
Chongyang Shen, ${ }^{\dagger}$ S Scott Bradford, ${ }^{\ddagger}$ Z Zhan Wang, ${ }^{\dagger} \S$ Yuanfang Huang, ${ }^{\dagger}$ Yulong Zhang, ${ }^{\S}$ \\ and Baoguo $\mathrm{Li}^{*}, \dagger$ \\ ${ }^{\dagger}$ Department of Soil and Water Sciences, China Agricultural University, Beijing 100193, China \\ ${ }^{\ddagger}$ U.S. Salinity Laboratory USDA, ARS, Riverside, California 92507-4617, United States \\ ${ }^{\S}$ College of Land and Environment, Shenyang Agricultural University, Shenyang, Liaoning 110866, China
}

\section{Supporting Information}

\begin{abstract}
The surface element integration technique was used to systematically study Derjaguin-Landau-Verwey-Overbeek (DLVO) interaction energies/forces between hollow spherical particles (HPs) and a planar surface or two intercepting half planes under different ionic strength conditions. The inner and outer spheres of HPs were concentric (CHP) or in point contact (PHP). In comparison to a solid particle, the attractive van der Waals interaction was reduced with increasing inner radius of the CHP, but the reduction effect was less significant for the CHP at smaller separation distance. Increasing the inner radius for CHP therefore reduced the depths of the secondary minima, but had minor influence on the energy barrier heights and depths of the primary minima. Consequently, increasing inner radius reduced the potential for CHP retention in secondary minima, whereas did not influence the retention in primary minima. For PHP these interaction energy parameters and colloid retention depended on the orientation of the inner sphere relative to interacting surface. In particular, the van der Waals attraction was significantly reduced at all

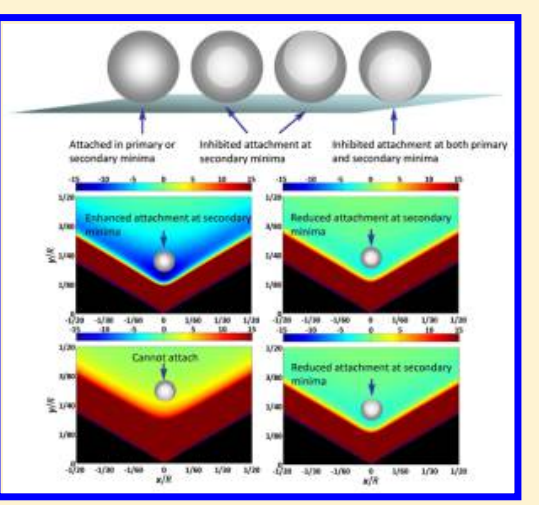
separation distances when the inner sphere was closest to the interacting surface, and this diminished retention in both secondary and primary minima. The PHP retention was similar to that of CHP when the inner sphere was farthest from the interaction surface. These orientation dependent interaction energies/forces resulted in directional bonds between PHPs and the formation of aggregates with contact points of the primary PHPs facing outward. The findings in this study have important implications for the design and utilization of HPs in soil remediation and colloid assembly.
\end{abstract}

\section{INTRODUCTION}

Hollow spherical particles (HPs) have unique structural, optical, and surface properties that have found wide applications as catalysts, drug delivery vehicles, photonic crystals, polymer fillers, and gas sensors. ${ }^{1,2}$ Fabrication of HPs has therefore drawn considerable attention in recent years. ${ }^{3-5}$ The hard templating method is most commonly used for fabrication of HPs. ${ }^{5,6}$ Hard templates such as silica colloidal particles and polymer spheres are typically coated with a layer of shell material, and hollow structures are obtained by selectively removing the templates. The compositions for HP shells include silica, carbon, polymer, metals, metal oxides, and complex compounds. ${ }^{5-7}$

The production and application of HPs will inevitably lead to their release into subsurface environments. Once released, HPs may encounter pollutants in soil or groundwater. Some HPs strongly adsorb pollutants such as toxic heavy metals and organic contaminants. ${ }^{8,9}$ The transport of adsorbed pollutants will be altered (diminish or enhance) by mobile HPs. Numerous studies have shown that the transport of pollutants in the soil can be significantly enhanced when they are associated with particles (i.e., the so-called colloid-facilitated transport of pollutants). ${ }^{10-13}$ Therefore, investigating transport of HPs in soil and aquifer material is essential to evaluate the risk of groundwater contaminated by the HP-associated pollutants.

The transport of particles within a porous medium is controlled by filtration mechanisms such as attachment and straining. ${ }^{14-18}$ The particle retention rate depends on transport of a particle from bulk flow to the vicinity of a collector surface and subsequent immobilization on the solid surface. ${ }^{19,20}$ Particle immobilization is determined by the balance of forces and torques that act on particles near the solid surface. ${ }^{10,16,21,22}$ The colloidal interaction forces between the particles and solid surfaces have typically been assumed to control particle retention by attachment. ${ }^{23-25}$ However, particle removal from solid surfaces may occur by diffusion and hydrodynamic torques in many instances ( $\mathrm{Li}$ et al., 2005; Shen et al., 2007, 2010; Bradford et al., 2011, 2013). ${ }^{16,26-29}$ The pore space geometry has a strong influence on the hydrodynamic forces

Received: July 8, 2017

Revised: $\quad$ September 7, 2017

Published: September 19, 2017 


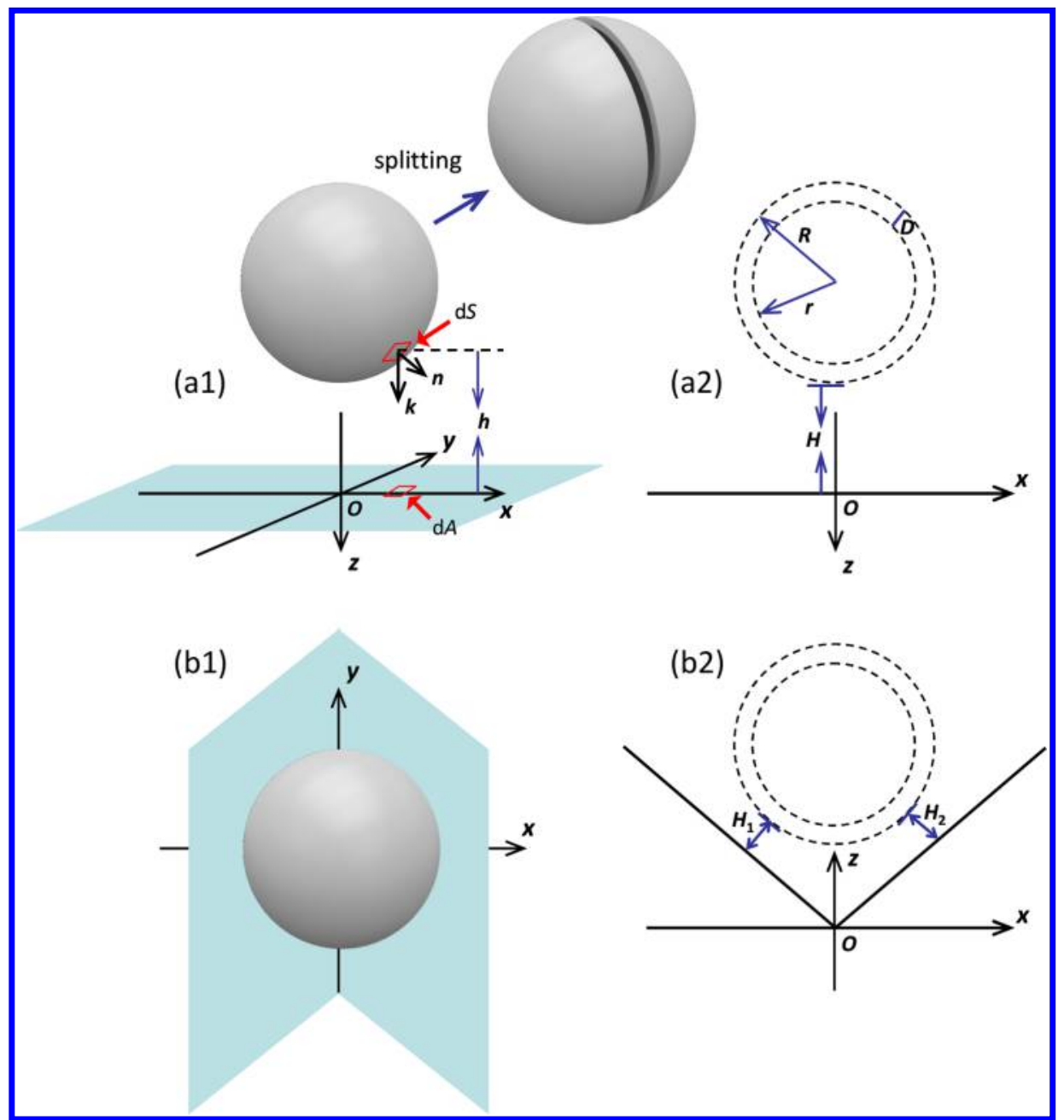

Figure 1. Coordinate systems used to describe a CHP interacting with (a) a planar surface or (b) two intersecting half planes. Parts (a2) and (b2) are plan view images of parts (a1) and (b1), respectively. $R$ and $r$ are radii of the outer and inner spheres of the CHP, respectively, $D$ is shell thickness of the CHP, $\mathrm{d} S$ is a differential area element on the CHP surface, $\mathbf{k}$ is the unit vector directed toward the positive $z$ axis, $\mathbf{n}$ is the outward unit normal to the particle surface, $\mathrm{d} A$ is the projected area of $\mathrm{d} S$ on the planar surface, $h$ is distance between $\mathrm{d} S$ and $\mathrm{d} A, H$ is separation distance between the CHP and the planar surface, and $H_{1}$ and $H_{2}$ are separation distances between the CHP and the left and right half planes, respectively.

and lever arms that act on particles, and grain-grain contacts have been found to serve as favorable locations for colloid retention. Particle retention in these locations has been referred to as surface straining. ${ }^{15,21,30-32}$

The colloidal interaction energy/force between a particle and a collector surface is quantitatively described by the DerjaguinLandau-Verwey-Overbeek (DLVO) theory. ${ }^{33}$ In this theory, the interaction energy is considered as the sum of van der Waals (VDW) and double layer (DL) interactions. The Derjaguin's approximation (DA) has commonly been used for calculating the VDW and DL interaction energies between a spherical particle and planar surface. However, the DA approach can only accurately estimate the interaction energies for large particles at small separation distances. Bhattacharjee and Elimelech ${ }^{34}$ developed the surface element integration (SEI) technique to overcome the limitations of the DA approach. In particular, the SEI approach can accurately estimate the interaction energy for any particle size (e.g., nanoparticles). ${ }^{34-36}$ Accurate estimation of interaction energies is not only of importance for theoretical understanding particle retention, but also for interpreting colloid assembly since the interaction energies also determine adhesive force between colloidal particles and accordingly the bond between them. ${ }^{37,38}$
Although the interaction energies between solid spherical particles (SPs) and collector surfaces have been extensively investigated, ${ }^{34,35,39,40}$ very limited research ${ }^{41-43}$ has been conducted to examine the interaction energies for HPs. Particularly, while the DL interaction energies between the HPs has been addressed, ${ }^{41,42}$ no published research has examined the total interaction energies (i.e., sum of VDW attraction, DL energy, and short-range repulsion) between the HPs and collector surfaces to date. This study therefore used the SEI technique to systematically examine the interaction energies/forces between the HPs and collector surfaces. We considered that the interiors of the HPs are spherical, and the inner and outer spheres of the HPs are concentric (designated as $\mathrm{CHP}$ ) or in point contact (PHP). Our calculations show that the mechanisms controlling the interactions between the HPs and collector surfaces are significantly different from those for SPs due to a reduction in van der Waals attraction. In addition, the interaction energies/forces between PHPs and surfaces are dependent on the orientations of the PHPs. Such orientation dependent interaction energies/forces can also lead to directional bonds between PHPs, which have important implications to designing and assembling of three-dimensional structures using colloidal particles. 


\section{THEORY}

Calculation of DLVO Interaction Energies between a HP and a Planar Surface. Figure 1a schematically illustrates the model system of a CHP interacting with a planar surface. The total interaction energy $\left(U_{\mathrm{T}}\right)$ between the CHP and the planar surface was considered as the sum of VDW attraction $\left(U_{\mathrm{VDW}}\right)$, DL interaction energy $\left(U_{\mathrm{DL}}\right)$, and short-range repulsion. The short-range repulsion was evaluated by determining the Born potential energy $\left(U_{\mathrm{BR}}\right) .^{10,25,44}$ The value of $U_{\mathrm{VDW}}$ was calculated as $U_{\mathrm{VDW}}^{\text {out }}-U_{\mathrm{VDW}}^{\text {in }}$, where $U_{\mathrm{VDW}}^{\text {out }}$ and $U_{\mathrm{VDW}}^{\mathrm{in}}$ are the VDW energies for the interaction of the planar surface with the outer and inner spheres, respectively. The outer or inner sphere represents a SP with the same composition as that of the CHP shell and the same size as that of the outer or inner sphere of the CHP. The DL and BR energies between the CHP and the planar surface are equal to those between the outer sphere and the planar surface (i.e., $U_{\mathrm{DL}}$ $=U_{\mathrm{DL}}^{\text {out }}$ and $\left.U_{\mathrm{BR}}=U_{\mathrm{BR}}^{\text {out }}\right)$. Hence, the expression used to calculate $U_{\mathrm{T}}$ is

$$
U_{\mathrm{T}}=U_{\mathrm{VDW}}^{\text {out }}-U_{\mathrm{VDW}}^{\text {in }}+U_{\mathrm{DL}}^{\text {out }}+U_{\mathrm{BR}}^{\text {out }}=U_{\mathrm{T}}^{\text {out }}-U_{\mathrm{VDW}}^{\text {in }}
$$

Note that the DL interaction is a surface force, so Equation 1 does not need to consider the influence of inner sphere on the $\mathrm{DL}$ interaction if the HP shell is close-grained (i.e., not permeable to ions). In addition, the influence of inner sphere on the Born repulsion is extremely small due to its rapid decrease with separation distance and thus it is also ignored in the calculations.

The SEI technique was used to calculate values of $U_{\mathrm{T}}^{\text {out }}$ and $U_{\mathrm{VDW}}^{\mathrm{in}}$. Briefly, the Cartesian coordinate system was adopted for the interaction configuration in Figure 1. The $x y$ plane of the coordinate system is oriented superposing the flat surface and the $z$ axis passes through the CHP center and faces away from the CHP. By discretizing the outer sphere surface into small area elements, the value of $U_{\mathrm{T}}^{\text {out }}$ can be calculated using the following equation ${ }^{45}$

$$
\begin{aligned}
U_{\mathrm{T}}^{\text {out }}(H)= & \sum_{S} E_{\mathrm{T}}(h) \mathbf{n} \cdot \mathbf{k} \mathrm{d} S \\
= & \sum_{A}\left(E_{\mathrm{T}}\left(H+R-\sqrt{R^{2}-\left(x^{2}+y^{2}\right)}\right)\right. \\
& \left.-E_{\mathrm{T}}\left(H+R+\sqrt{R^{2}-\left(x^{2}+y^{2}\right)}\right)\right) \mathrm{d} A
\end{aligned}
$$

where $H$ is the separation distance between the outer sphere and the planar surface, $E_{\mathrm{T}}$ is differential interaction energy between an area element $\mathrm{d} S$ on the outer sphere surface and the planar surface, $h$ is local separation distance between the element $\mathrm{d} S$ and the planar surface, $\mathbf{n}$ is unit outward normal to the outer sphere surface, $\mathbf{k}$ is unit vector along the positive $z$ direction, $S$ is the total surface area of the outer sphere, $A$ is total projected area of the outer sphere surface on the planar surface, $R$ is radius of the outer sphere, and $\mathrm{d} A$ is the projected area of $\mathrm{d} S$ on the planar surface.

The differential interaction energy $E_{\mathrm{T}}$ was calculated by adding $E_{\mathrm{VDW}}, E_{\mathrm{DL}}$, and $E_{\mathrm{BR}}$. The expressions used to calculate $E_{\mathrm{VDW}}, E_{\mathrm{DL}}$, and $E_{\mathrm{BR}}$ are following ${ }^{46-48}$

$$
E_{\mathrm{VDW}}(h)=-\frac{A_{\mathrm{H}}}{12 \pi h^{2}}
$$

$$
\begin{aligned}
& E_{\mathrm{DL}}(h)=\frac{\varepsilon \varepsilon_{0} \kappa}{2}\left[\left(\psi_{\mathrm{p}}^{2}+\psi_{\mathrm{c}}^{2}\right)(1-\operatorname{coth} \kappa h)+\frac{2 \psi_{\mathrm{p}} \psi_{\mathrm{c}}}{\sinh \kappa h}\right] \\
& E_{\mathrm{BR}}(h)=\frac{A_{\mathrm{H}} H_{0}^{6}}{48 \pi h^{8}}
\end{aligned}
$$

where $A_{\mathrm{H}}$ is the Hamaker constant of the interacting media, $\varepsilon_{0}$ is dielectric permittivity of vacuum, $\varepsilon$ is dielectric constant of water, $\kappa$ is inverse Debye screening length, $\psi_{\mathrm{p}}$ and $\psi_{\mathrm{c}}$ are surface potentials of the outer sphere and the planar surface, respectively, and $H_{0}$ is the minimum separation distance, taken as $0.158 \mathrm{~nm} .{ }^{49}$ Note that a uniform distribution of surface charge and constant surface potential in the interaction system were assumed by using eq $4 .{ }^{44}$

The procedure used to calculate the $U_{\mathrm{VDW}}^{\text {in }}$ is similar to that for calculating $U_{\mathrm{VDW}}^{\text {out }}$, which is written as

$$
\begin{aligned}
U_{\mathrm{VDW}}^{\mathrm{in}}(H)= & \sum_{A}\left(E_{\mathrm{VDW}}\left(H+R-\sqrt{r^{2}-\left(x^{2}+y^{2}\right)}\right)\right. \\
& \left.-E_{\mathrm{VDW}}\left(H+R+\sqrt{r^{2}-\left(x^{2}+y^{2}\right)}\right)\right) \mathrm{d} A
\end{aligned}
$$

where $r$ is the radius of the inner sphere of the CHP.

For the interaction of a PHP with a planar surface, various orientations are possible (see Figure S1 of Supporting Information). Only two limiting cases were considered: the contact point of the inner and outer spheres of the PHP is closest to or farthest from the planar surface. The former and later interaction orientations were denoted as CPS and FPS, respectively. The PHP can stably interact on the planar surface in the two limiting orientations. The PHP on the planar surface in other orientations will rotate due to the presence of a net torque until one of the limiting orientations is formed. ${ }^{50}$ The procedure used to calculate the interaction energy between the PHP and the collector surface is similar to that for the CHP as shown above. However, the equation used to calculate the value of $U_{\mathrm{VDW}}^{\mathrm{in}}$ in the CPS has to be

$$
\begin{aligned}
U_{\mathrm{VDW}}^{\mathrm{in}}(H)= & \sum_{A}\left(E_{\mathrm{VDW}}\left(H+r-\sqrt{r^{2}-\left(x^{2}+y^{2}\right)}\right)\right. \\
& \left.-E_{\mathrm{VDW}}\left(H+r+\sqrt{r^{2}-\left(x^{2}+y^{2}\right)}\right)\right) \mathrm{d} A
\end{aligned}
$$

The equation for calculating the value of $U_{\mathrm{VDW}}^{\mathrm{in}}$ in the FPS is

$$
\begin{aligned}
U_{\mathrm{VDW}}^{\text {in }}(H)= & \sum_{A}\left(E_{\mathrm{VDW}}\left(H+2 R-r-\sqrt{r^{2}-\left(x^{2}+y^{2}\right)}\right)\right. \\
& \left.-\mathrm{E}_{\mathrm{VDW}}\left(H+2 R-r+\sqrt{r^{2}-\left(x^{2}+y^{2}\right)}\right)\right) \mathrm{d} A
\end{aligned}
$$

Calculation of Adhesive and Hydrodynamic Torques. When only VDW and DL interaction energies are considered, a particle that overcomes the energy barrier will be irreversibly attached in an infinitely deep primary minimum. However, colloid interactions may be reversible in the presence of shortrange Born repulsion that produces a finite depth of the primary minimum. Therefore, the attachment of a particle in the primary minimum is not only dependent on the maximum energy barrier, but also on the balance between adhesive and hydrodynamic torques that act on the particle when flow is present. $^{22-24,51}$ 


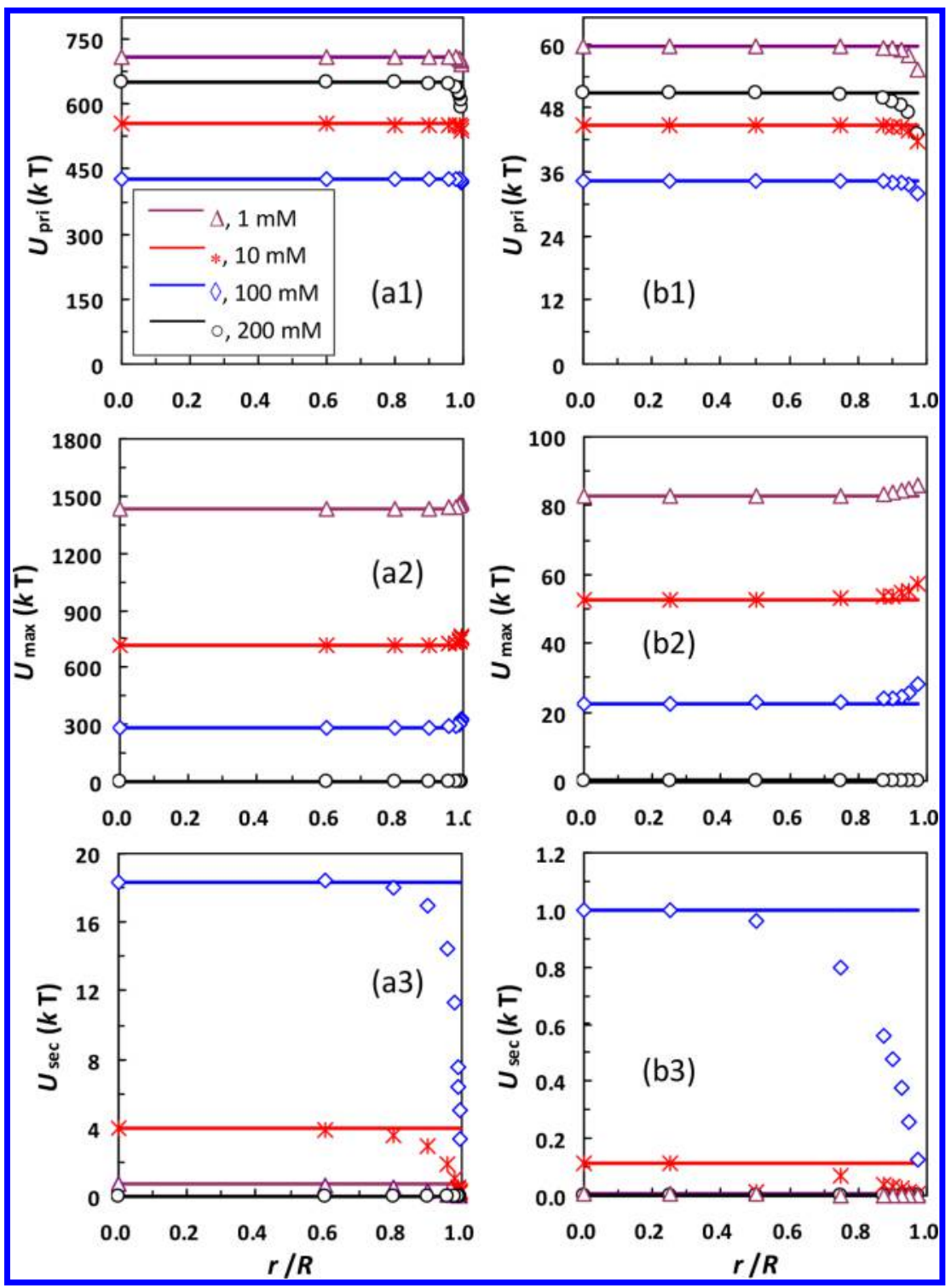

Figure 2. Calculated primary minimum depth $\left(U_{\text {pri }}\right)$, maximum energy barrier $\left(U_{\max }\right)$, and secondary minimum depth $\left(U_{\text {sec }}\right)$ for $(\mathrm{a}) 1000$ and $(\mathrm{b}) 80$ $\mathrm{nm}$ silica CHPs with different inner radii under various IS conditions. The values of $U_{\text {pri, }} U_{\max }$ and $U_{\text {sec }}$ for the 1000 and $80 \mathrm{~nm}$ silica SPs (solid lines) were also shown.

The detailed procedure for determining the adhesive and hydrodynamic torques for particles on smooth, planar surfaces has been previously presented in the literature. ${ }^{28,52}$ Briefly, the adhesive torque $\left(T_{\mathrm{A}}\right)$ is represented by the adhesive force $\left(F_{\mathrm{A}}\right)$ acting on a lever arm $l_{c}$

$$
T_{\mathrm{A}}=F_{\mathrm{A}} l_{\mathrm{c}}
$$

The value of $F_{\mathrm{A}}$ is taken as the maximum attraction which is obtained through calculating the derivative of DLVO interaction energies with respect to $H^{53,54}$ The lever arm is equal to the radius of particle surface contact area $(\omega)$ due to deformation of the attached HP. As there is no expression available for calculating the value of $\omega$ for the HP to date, the equation developed for the SP was used for the calculations, which is given as ${ }^{26,55}$

$$
\omega=\left(\frac{4 F_{\mathrm{A}} R}{E}\right)^{1 / 3}
$$

where $E$ is elastic interaction constant. When the shell of the $\mathrm{HP}$ is thick, the deformation of the HP is similar to that of the SP and, hence, the use of eq 10 for estimating the values of $\omega$ is accurate. The HP will be deformed to a greater extent than the SP for a given attractive force when the shell of the HP is very thin. In these cases, eq 10 provides a lower bound of $\omega$ for the HP.

The hydrodynamic drag torque $\left(T_{\mathrm{H}}\right)$ that acts on the HP in the vicinity of a planar surface is written as ${ }^{52}$

$$
T_{\mathrm{H}}=1.4 R F_{\mathrm{D}}
$$

where $F_{D}$ is the drag force. The equation used to calculate $F_{D}$ is $^{22,29}$

$$
F_{\mathrm{D}}=1.7007 \cdot 6 \pi \mu R V
$$

where $\mu$ is viscosity of water and $V$ is the relative velocity between the fluid and the HP at the center of the HP. A typical rate of groundwater flow $(4 \mathrm{~m} / \mathrm{d})$ in coarse textured porous media was assumed for $V$ to calculate the value of $F_{\mathrm{D}}$ and $T_{\mathrm{H}}{ }^{36}$ 


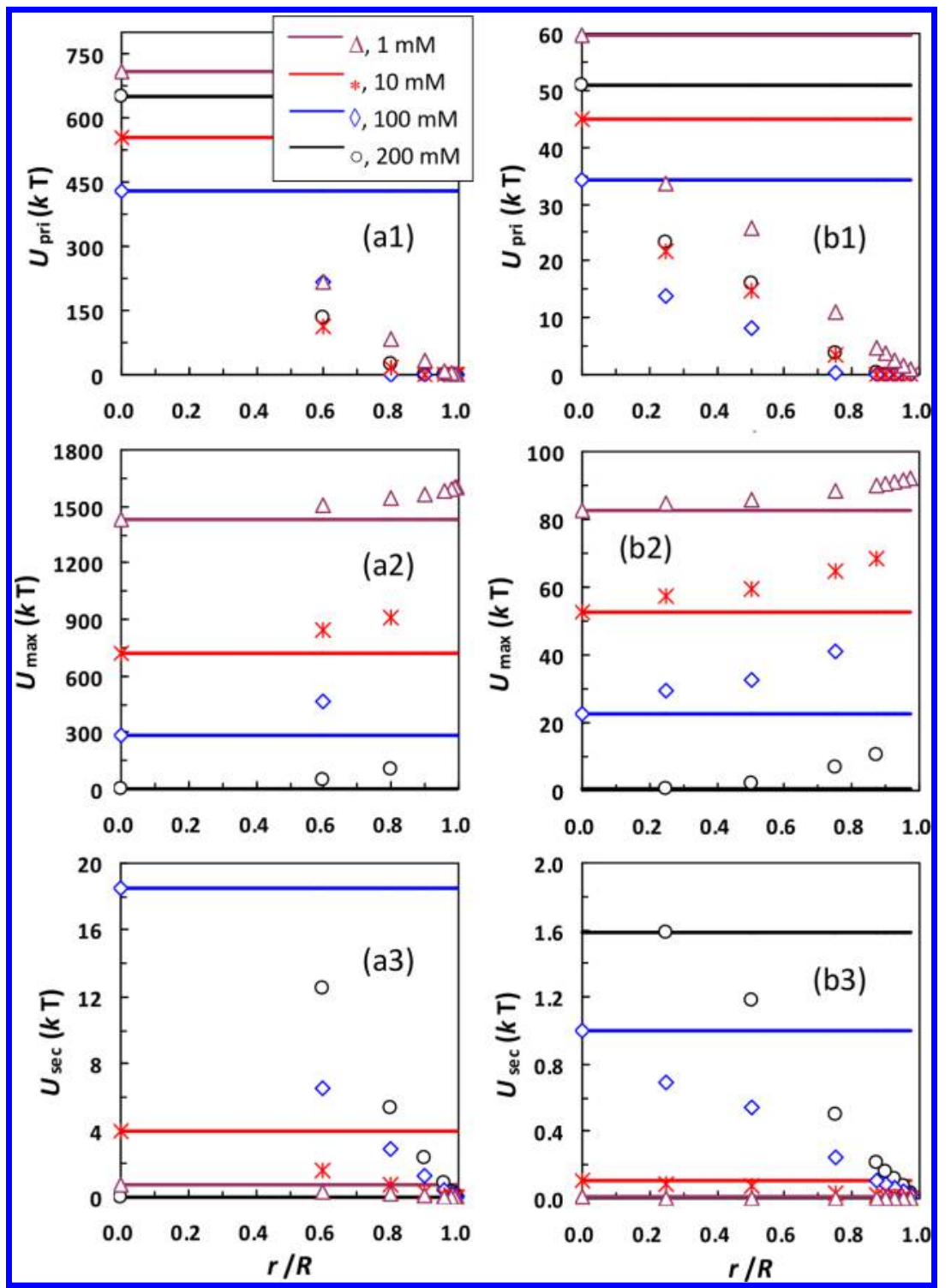

Figure 3. Calculated values of $U_{\text {pri }} U_{\max }$ and $U_{\text {sec }}$ for the (a) 1000 and (b) $80 \mathrm{~nm}$ silica PHPs with different inner radii in the CPS orientation under various IS conditions. The values of $U_{\text {pri }}, U_{\max }$, and $U_{\text {sec }}$ for the 1000 and $80 \mathrm{~nm}$ silica SPs (solid lines) were also shown.

\section{RESULTS AND DISCUSSION}

For theoretical calculations, the shell composition of the HP was assumed to be silica. The outer diameters of the HPs were taken as 1000 and $80 \mathrm{~nm}$. The collector surface was assumed to be planar, with the same properties as those of quartz sand surface. The DLVO interaction energies/forces between the $\mathrm{HP}$ and the planar surface were calculated for the HPs at an ionic strength (IS) of $1,10,100$, and $200 \mathrm{mM} \mathrm{NaCl}$. The zeta potentials of silica HPs and sand in $\mathrm{NaCl}$ at different IS were taken from Wang et al. ${ }^{56,57}$ and Shen et al. ${ }^{27}$ (see Table S1 of Supporting Information). A value of $6.3 \times 10^{-21} \mathrm{~J}$ was chosen as the Hamaker constant for the silica-water-quartz system. $^{57,58}$

Interaction between a CHP and a Planar Surface. DLVO interaction energy profiles (IEPs) for the 1000 and 80 $\mathrm{nm}$ silica CHPs and SPs with different inner radii were calculated and shown in Figure S2 of Supporting Information for various IS conditions. The shape of the IEP for the 1000 or $80 \mathrm{~nm}$ CHP is similar to that for its corresponding SP (i.e., with the same size as that of the CHP) at a given IS. Specifically, the
IEPs of both CHPs and SPs are characterized by a deep primary energy well at a small separation distance, a maximum energy barrier, and a shallow secondary energy well at a larger distance (denoted as type I IEP) when IS $\leq 100 \mathrm{mM}$. The shallow secondary energy wells in the IEPs for the two CHPs are highlighted in Figure S3 of Supporting Information. At 200 $\mathrm{mM}$, both energy barrier and secondary minimum disappear and only the primary minimum exists in the IEPs of the CHPs and SPs (type II). The two types of IEPs have been widely reported in the literature. ${ }^{59-61}$

Figure 2 presents the primary minimum depth $\left(U_{\text {pri }}\right)$, maximum energy barrier $\left(U_{\max }\right)$, and secondary minimum depth $\left(U_{\text {sec }}\right)$ from the IEPs in Figure S2 of Supporting Information as a function of inner radius of the 1000 or $80 \mathrm{~nm}$ $\mathrm{CHP}$. Increasing the CHP inner radius decrease the value of $U_{\text {pri }}$ due to a reduction in VDW attraction. However, even if the CHP shell is very thin, the reduction of $U_{\text {pri }}$ is small compared to its corresponding SP, demonstrating that the value of $U_{\text {pri }}$ between a SP and a surface is mainly determined by a small fraction of the SP closet to the surface. The value of $U_{\max }$ 

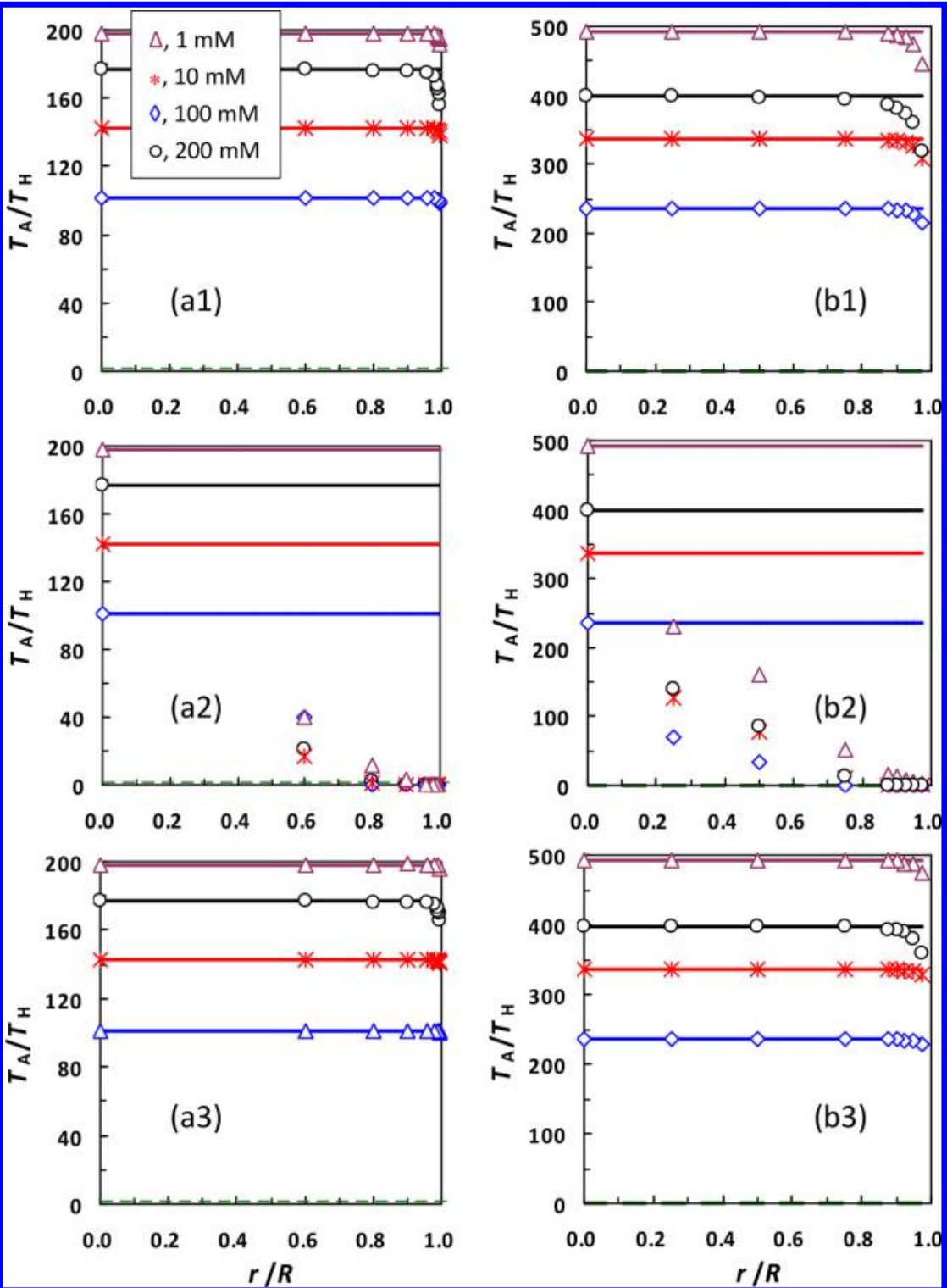

Figure 4. Calculated adhesive torque $\left(T_{\mathrm{A}}\right)$ normalized by a hydrodynamic drag torque $\left(T_{\mathrm{H}}\right)$ as a function of shell thickness for the (a) 1000 and $(\mathrm{b})$ $80 \mathrm{~nm}$ silica HPs (1, CHPs; 2, PHPs in CPS orientation; 3, PHPs in FPS orientation) under various IS conditions. Soil lines represent values of $T_{\mathrm{A}} /$ $T_{\mathrm{H}}$ for the 1000 and $80 \mathrm{~nm}$ silica SPs, and dashed line represents $T_{\mathrm{A}} / T_{\mathrm{H}}=1$.

increases very slightly with increasing inner radius for a similar reason. These results illustrate that the attachment/detachment of the CHP at/from primary minima is similar to that of the corresponding SP. In contrast, the value of $U_{\text {sec }}$ decreases significantly with an increase in the CHP inner radius. For example, the value of $U_{\text {sec }}$ decreases from $18.4 k T$ ( $k$ is Boltzmann constant and $\mathrm{T}$ is absolute temperature) to $3.4 \mathrm{kT}$, with an increase of inner radius of the $1000 \mathrm{~nm}$ CHP from 0 to $498 \mathrm{~nm}$ at $100 \mathrm{mM}$. The $1000 \mathrm{~nm} \mathrm{CHP} \mathrm{can} \mathrm{readily} \mathrm{escape} \mathrm{this}$ shallow secondary minimum by Brownian diffusion and/or hydrodynamic shear. Hence, the secondary minimum attachment of CHPs with thin shells is inhibited.

Interaction between a PHP and a Planar Surface. Figure S4 of the Supporting Information presents calculated IEPs for the 1000 or $80 \mathrm{~nm}$ silica PHP with different inner radii in CPS orientation under various IS conditions. In addition to the aforementioned two types of IEPs, two more types of IEP are present for the PHPs with thin shells. Specifically, both primary and secondary minima disappear from the IEP and the interaction energy decreases monotonically with increasing separation distance (e.g., when $2 r=996 \mathrm{~nm}$ and IS $=1 \mathrm{mM}$ ) (type III). This type of IEP indicates that the PHP experience repulsive force at all separation distances, so the PHP cannot be attached on the collector surface in the CPS orientation. For the other type of IEP (type IV), the primary minimum is absent and only a shallow attractive well (i.e., the secondary minimum) is present at a large distance (e.g., when $2 r=996 \mathrm{~nm}$ and IS $\geq$ $10 \mathrm{mM}$ in Figure S5 of Supporting Information).

Figure 3 presents calculated values of $U_{\text {pri }}, U_{\max }$ and $U_{\text {sec }}$ as a function of inner radius for the 1000 or $80 \mathrm{~nm}$ silica PHP in the CPS orientation at different IS conditions. Both values of $U_{\text {pri }}$ and $U_{\text {sec }}$ decrease with increasing inner radius because of the decrease in $U_{\mathrm{VDW}}$. In particular, the primary minimum disappears from the IEPs (i.e., $U_{\text {pri }}=0$ ) for the $1000 \mathrm{~nm}$ PHP with $r \geq 450 \mathrm{~nm}$ and for the $80 \mathrm{~nm}$ PHP with $r \geq 36 \mathrm{~nm}$ (i.e., $r / R \geq 0.9$ ) when $10 \mathrm{mM} \leq \mathrm{IS} \leq 200 \mathrm{mM}$. Moreover, the values of $U_{\text {sec }}$ are small for the $1000 \mathrm{~nm}(<0.8 k T)$ and $80 \mathrm{~nm}$ $(<0.2 k T)$ PHPs under the aforementioned conditions. These 
$U_{\text {sec }}$ are significantly smaller than the average kinetic energy of a diffusing colloid $(1.5 k T)$, and PHPs therefore cannot be immobilized in these shallow attractive wells. Hence, PHPs cannot be attached on collector surfaces in either primary or secondary minima in these cases.

It is interesting to note that while only the primary minimum is present in the IEP (i.e., type II) for the 1000 and $80 \mathrm{~nm} \mathrm{SPs}$ at $200 \mathrm{mM}$, the IEP becomes type I for $0<r / R<0.9$ and type IV for $0.9 \leq r / R \leq 1$ (see Figure 3) for the PHPs in the CPS orientation under these same conditions. Hence, the attachment and detachment mechanisms of PHPs are completely different from those of SPs. Specifically, the SPs are favored to be attached in a primary minima at $200 \mathrm{mM}$ since no energy barrier is present. In addition, the primary minimum attachment is irreversible to the reduction of IS because the primary minimum depth and, accordingly, the adhesion acting on the SPs at the energy well increases with decreasing IS. In contrast, the energy barrier and secondary minima are significant for PHPs when $0<r / R<0.9$ and the IS $=200 \mathrm{mM}$. Hence, the PHPs are favored to be attached in a secondary minimum in the CPS orientation. The attachment in the secondary minima is reversible with a reduction in IS, as the secondary minimum depth decreases with decreasing IS. ${ }^{60-62}$ PHPs cannot be attached in the CPS orientation when $0.9 \leq r / R \leq 1$ because the secondary energy wells of the type IV IEP are very shallow (see Figure S5 of the Supporting Information).

Figure S6 of the Supporting Information presents calculated $U_{\text {pri }} U_{\max }$ and $U_{\text {sec }}$ as a function of inner radius for the 1000 and $80 \mathrm{~nm}$ silica PHPs in FPS orientation under various IS conditions. Similar to the interaction of CHPs with the collector surface, increasing the inner radii of the PHPs only slightly decreases and increases the values of $U_{\text {pri }}$ and $U_{\max }$ respectively. Hence, the attachment and detachment of the PHPs in the FPS orientation are similar to those of SPs and CHPs. In contrast, the value of $U_{\text {sec }}$ decreases rapidly with $r$ for the PHPs at large ratios of $r / R$ (e.g., $r / R \geq 0.9$ for the $1000 \mathrm{~nm}$ $\mathrm{PHP})$. Accordingly, attachment in a secondary minimum is inhibited for PHPs in these cases. Interestingly, the results in Figures 2, 3, and S6 of the Supporting Information consistently show that attachment in the secondary minimum is always inhibited for a HP with large inner radius, irrespective of the location of the inner sphere in the HP.

It is worthwhile mentioning that the aforementioned results for the HP attachment and detachment were obtained through comparing colloidal interaction energies and Brownian diffusion. If flow is present, the HPs at the energy minima also experience hydrodynamic shear forces. The HPs in secondary minima are susceptible to hydrodynamic shear because secondary minima are located at a certain distance from collector surfaces. These HPs will be swept away from the collector surfaces when the applied hydrodynamic torque exceeds the resisting adhesive torque. The HPs may also translate and rotate along the collector surfaces until they are immobilized in regions with reduced flow velocity, and altered lever arms (e.g., grain-grain contacts and microscopic roughness locations). Colloid filtration theory (CFT) neglects the influence of hydrodynamic drag on the particles and assumes that particles will be firmly attached in a primary minimum once they overcome the energy barrier. ${ }^{10}$ Indeed, Figure 4 shows that adhesive torques that the SPs experience are more than 2 orders of magnitude larger than the hydrodynamic torques for the 1000 and $80 \mathrm{~nm}$ SPs. Adhesive torques are also significantly larger than the hydrodynamic torques for the 1000 and $80 \mathrm{~nm} \mathrm{CHPs} \mathrm{and} \mathrm{PHPs} \mathrm{in} \mathrm{the} \mathrm{FPS}$ orientation. Consequently, attachment in the primary minima is not influenced by the hydrodynamic drag in these cases. In contrast, the adhesive torque rapidly decreases with the inner radius of the 1000 or $80 \mathrm{~nm}$ PHP in the CPS orientation, which is even smaller than the hydrodynamic torque at $r / R \geq 0.8$. Therefore, PHP with thin shells cannot be attached in primary minima in the CPS orientation under typical groundwater flow conditions.

The perfect sink model has been used in CFT as the boundary condition at a collector surface to derive the equation of single collector contact efficiency. ${ }^{18,63,64}$ In the perfect sink model, the primary minimum is assumed to be an infinite energy sink and all particles arriving at the collector surface are irreversibly consumed in the energy sink by a very fast immobilization reaction. ${ }^{10}$ This assumption is reasonable for SPs due to the dominance of the adhesion torque over the hydrodynamic torque. However, Shen et al. ${ }^{65}$ and Rasmuson et al. ${ }^{66}$ showed that the presence of nanoscale protruding asperities on collector surfaces can cause the breakdown of the perfect sink model because the nanoscale asperities can significantly decrease the adhesive torques of the SPs at primary minima to be below the hydrodynamic torques. Our study further shows that the perfect sink model is not applicable for deposition of the HPs even if the collector surface is perfectly smooth.

Although the result of $T_{\mathrm{A}}<T_{\mathrm{H}}$ was obtained for the silica HPs with negatively charged surfaces, Figure S7 of the Supporting Information show that the adhesive torque can become smaller than the hydrodynamic drag torque even for zerovalent iron (ZVI) PHPs in the CPS orientation at large ratios of $r / R$. This result indicates that ZVI PHPs cannot be attached in the CPS orientation under typical groundwater flow conditions. Although the ZVI PHP can be attached in a primary minimum in the FPS orientation, the PHPs, as will be shown later in the paper, are favored to be aggregated with the contact point of the inner and outer spheres facing outward (see Figure S8, Supporting Information). Such aggregates could have great mobility in the subsurface environments since they are not favored to be attached on collector surfaces (especially in the presence of flow) and they experience lower gravitational force and greater buoyancy forces in solutions compared to SPs.

ZVI particles have been shown to be very effective for transformation and detoxification of various environmental pollutants. ${ }^{67-69}$ However, application of the ZVI SPs for in situ remediation of contaminated soil is very limited due to their very low mobility in the soil. ${ }^{70-73}$ Although modification of the ZVI particles with polymers can enhance their transport in the soil, covering the particle surfaces with polymers could also decrease their capacities for pollutant removal. ${ }^{74}$ Alternatively, our study provides a novel way (i.e., fabrication of ZVI particles with hollow structures) to enhance the mobility of the ZVI particles in soil without using polymers.

Interaction between a HP and Two Intersecting Half Planes. The above results were obtained by considering the interaction of a HP with only one collector surface. When a particle is retained in a porous medium by straining, the particle interacts with multiple collector surfaces. Particularly, the interaction of a particle with only two collector surfaces is termed as wedging, which is a dominant process of straining. ${ }^{31,32,75}$ To interpret the wedging of a CHP and $\mathrm{PHP}$, the interaction forces between the CHP or PHP and two intersecting half planes were calculated (i.e., Figure $1 \mathrm{~b}$ and 

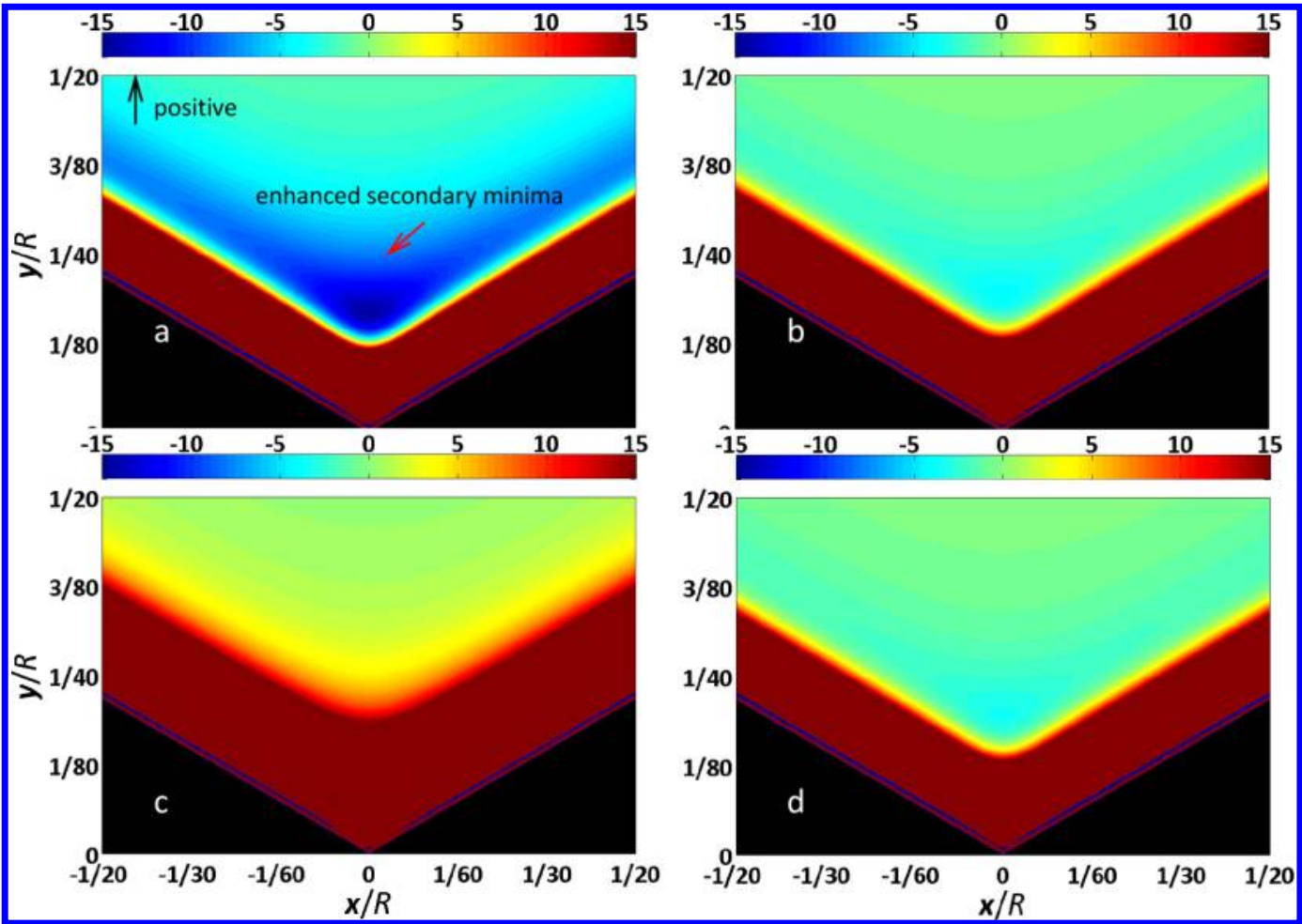

Figure 5. Maps of DLVO force component along the $y$-direction $\left(F_{y}\right)$ normalized by a hydrodynamic drag force $\left(F_{\mathrm{D}}\right)$ for interactions between a $1000 \mathrm{~nm}$ silica particle (a, SP; b, CHP; c, PHP in FTV orientation; d, PHP in FAV orientation) and two intersecting half planes with an angle of $120^{\circ}$ on the $x-y$ plane at $z=0$ at $100 \mathrm{mM}$. The black regions indicate the locations where the interaction force could not be determined due to physical overlap between the colloid and the concave surface. Positive values represent the components of DLVO interaction force along positive $y$ direction. The inner radii of the CHP and PHP are $498 \mathrm{~nm}$.

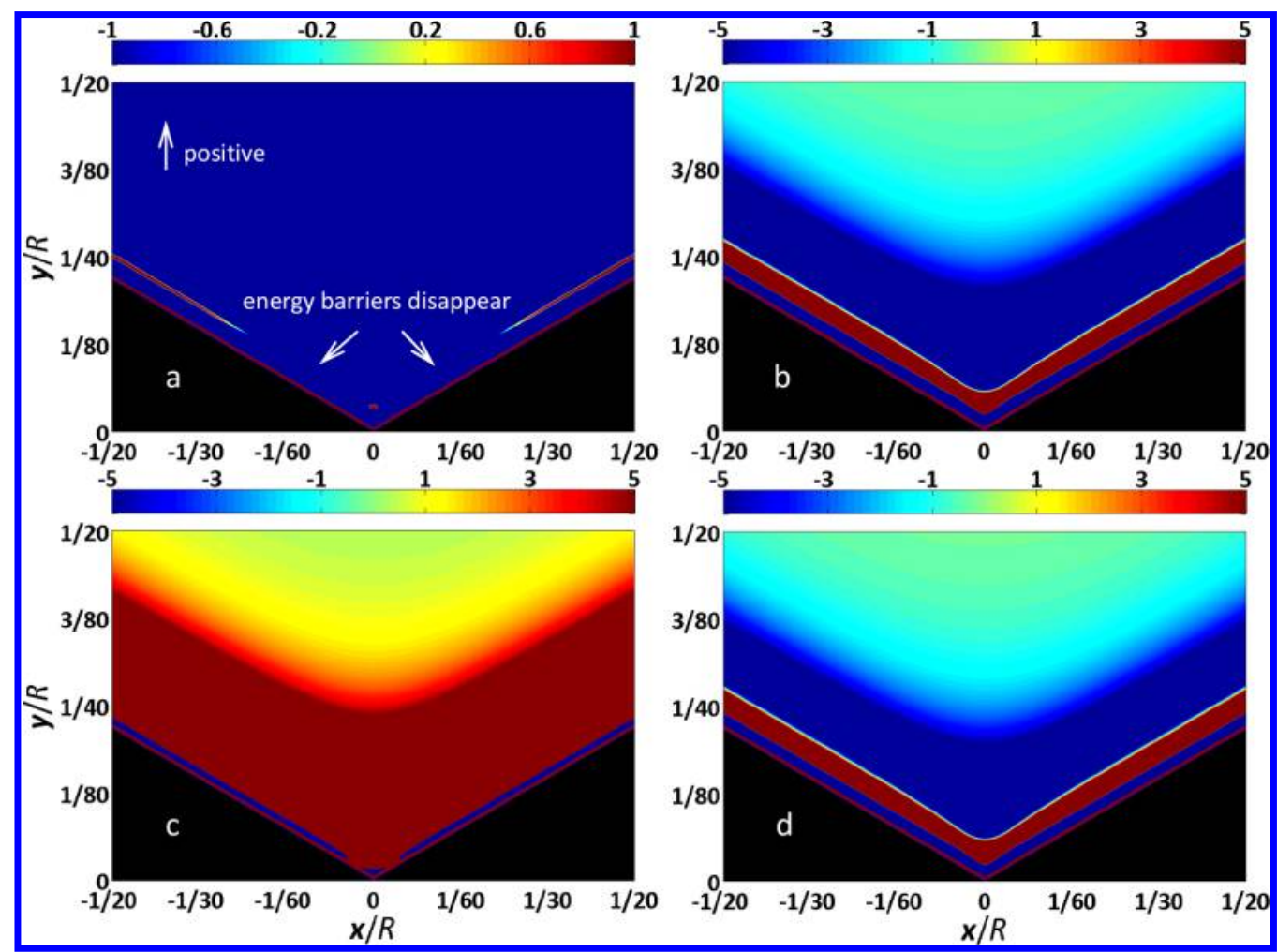

Figure 6. Maps of component of DLVO force along the $y$-direction $\left(F_{y}\right)$ normalized by a hydrodynamic drag force $\left(F_{\mathrm{D}}\right)$ for interactions between a $1000 \mathrm{~nm}$ silica particle (a, SP; b, CHP; c, PHP in FTV orientation; d, PHP in FAV orientation) and two intersecting half planes with an angle of $120^{\circ}$ on the $x-y$ plane at $z=0$ at $200 \mathrm{mM}$. All simulation conditions are consistent with those in Figure 5 except the interaction force scales. The inner radii of the $\mathrm{CHP}$ and $\mathrm{PHP}$ are $498 \mathrm{~nm}$. 
Figure S1, Supporting Information). The two intersecting half planes can also be used to represent wedge-like depressions on rough collector surfaces. ${ }^{76}$ The DLVO interaction force was calculated for these interaction configurations instead of estimating the interaction energy because it is more convenient for interpreting mobilization of the HPs within the concave surface. Note that various orientations are present for interaction of the PHP with the two intersecting half planes. Two typical cases were considered for the PHP in this study: the line through the centers of the inner and outer spheres and the interception line of the two half planes cross each other and the contact point of the inner and outer spheres of the PHP faces toward or away from the vertex of the two intercepting half planes (see Figure S1e,f of the Supporting Information). The former and latter interaction orientations were denoted as FTV and FAV, respectively. Detailed procedure for calculating the interaction forces for the CHP and PHP has been given in the Supporting Information.

Figure 5 presents maps of the DLVO force component along the $y$-direction $\left(F_{y}\right)$ normalized by $F_{\mathrm{D}}$ for interactions between a $1000 \mathrm{~nm}$ silica SP, CHP, or PHP and the two intersecting half planes with an angle of $120^{\circ}$ on the $x-y$ plane at $z=0$ in Figure $1 \mathrm{~b}$ and Figure S1e,f of the Supporting Information at $100 \mathrm{mM}$. The inner radii of the CHP and PHP are $498 \mathrm{~nm}$. The black regions indicate the locations where the interaction force could not be determined due to physical overlap between the colloid and the concave surface. Figure 5 shows that the secondary minimum force is significantly enhanced for the SPs in the area above the energy barrier (i.e., the red strips), indicating that the SPs are favored to be retained in the enhanced secondary minima at $100 \mathrm{mM}$. In contrast, the secondary minimum wells are very shallow in the concave area for the CHP and PHP in FAV orientation, which even disappear for the PHP in FTV orientation. Therefore, the HPs cannot be retained in the concave area via secondary-minimum association at this IS.

Figure 6 shows maps of $F_{y}$ normalized by the $F_{D}$ for the same interaction configurations as those in Figure 5 at a different IS (i.e., $200 \mathrm{mM}$ ). The energy barrier is very small for the silica SP, which even completely disappears in the area close to the vertex of the two intercepting half planes (denoted as VAREA). Hence, the silica SP is favored to be retained at the primary minima in the VAREA. For the silica CHP and PHP in FAV orientation, both energy barriers and secondary minima are significant, indicating that retention in the secondary minima is preferred in these two cases. For the PHP in FTV orientation, the energy barrier is further increased and the secondary minimum disappears in the concave region. Accordingly, the PHP cannot be retained in either primary or secondary minimum in the FTV orientation when IS $=200 \mathrm{mM}$. The results in Figures 5 and 6 indicate that the retention mechanisms of the HPs are completely different from those of the SPs in the grain-to-grain contact area. In addition, the release of the retained HPs is also different from that of the SPs. For example, the SPs retained in the VAREA via primaryminimum association in Figure 6a are irreversible to reduction of solution IS since the detachment energy barrier from primary minimum increases with decreasing IS (see Figure S9, Supporting Information). Conversely, the CHPs and PHPs retained in the secondary minima at $200 \mathrm{mM}$ in Figure $6 \mathrm{~b}, \mathrm{~d}$ can be released by reducing the IS to eliminate the energy wells. Therefore, while the retained SPs in grain-grain contacts cannot be released by merely reducing IS unless conducting dissection experiments to destroy the pore structure, the retained HPs are reversible to IS reduction.

Interaction between Two HPs. The aforementioned results show different attachment and detachment behavior for the HPs compared to those of SPs. The aggregation between two HPs and subsequent disaggregation are also different from those of SPs. For example, Figure 7 presents

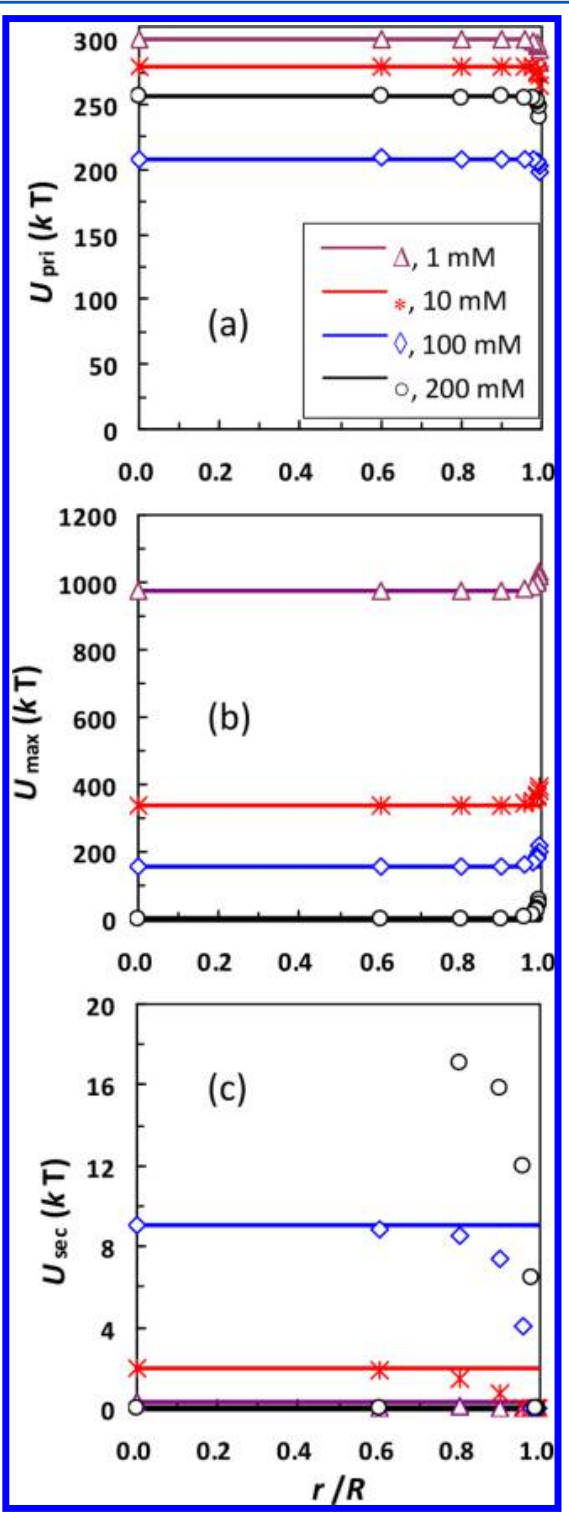

Figure 7. Calculated values of $U_{\text {pri }} U_{\max }$ and $U_{\text {sec }}$ for the interaction between two $1000 \mathrm{~nm}$ silica CHPs with different inner radii at different ISs. The values of $U_{\text {prij }} U_{\max }$ and $U_{\text {sec }}$ for the interaction between two $1000 \mathrm{~nm}$ silica SPs (solid lines) were also shown.

calculated values of $U_{\text {pri, }} U_{\max }$ and $U_{\text {sec }}$ for the interactions between two $1000 \mathrm{~nm}$ silica SPs or two CHPs with different inner radii under different IS conditions. Details about calculation of the interaction energies between two HPs have been given in the Supporting Information. Figure 7 shows that only the primary minimum, and therefore favorable aggregation, is present between the two interacting SPs at $200 \mathrm{mM}$. For the interaction between two CHPs, both the energy barrier and secondary minimum appear at this IS when $r / R \geq 0.8$. Therefore, aggregation could occur in a secondary minimum 


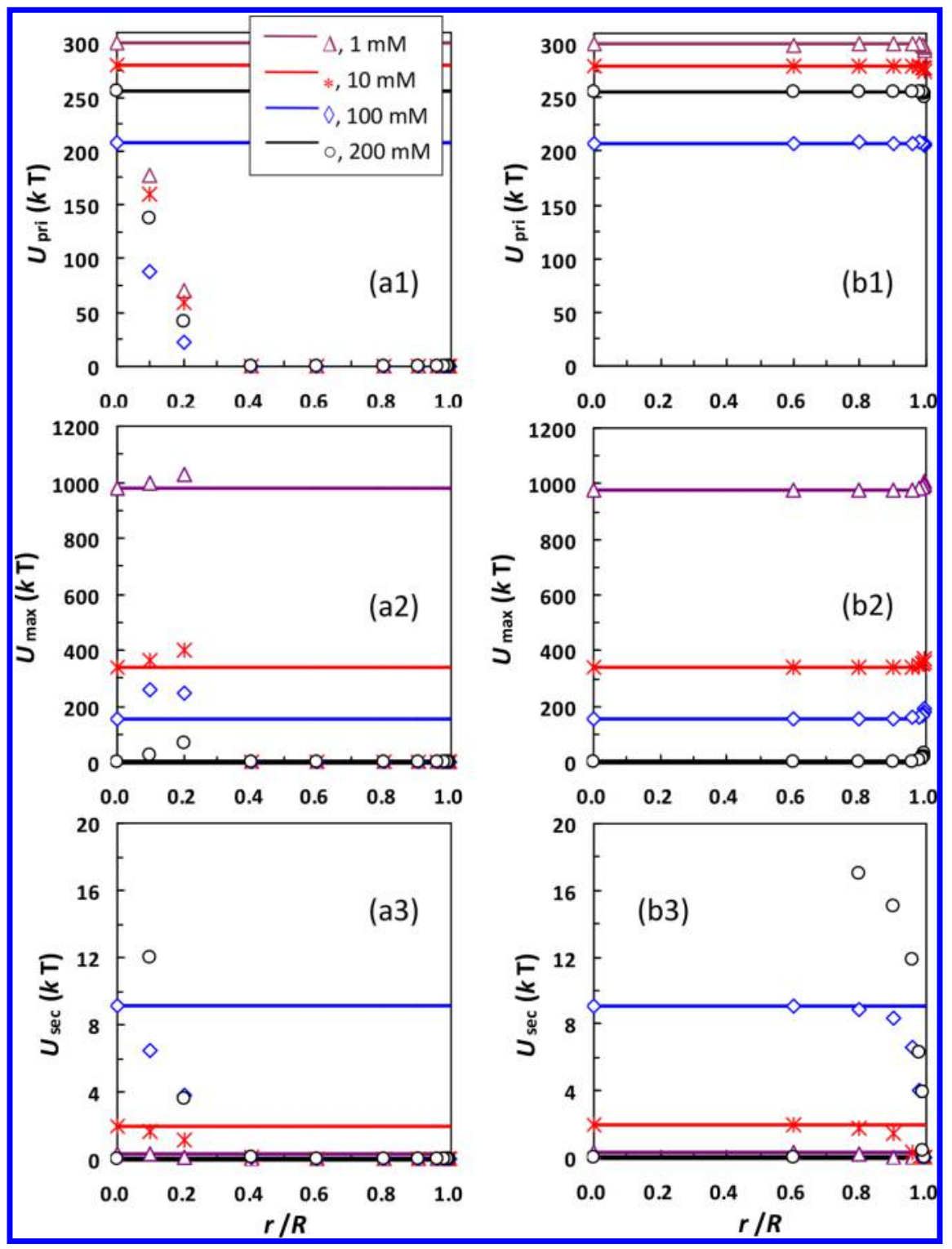

Figure 8. Calculated values of $U_{\text {pri }} U_{\max }$, and $U_{\text {sec }}$ for the interaction between two $1000 \mathrm{~nm}$ silica PHPs with different inner radii in (a) TWD and (b) AWY orientations at different ISs. The values of $U_{\text {pri }} U_{\max }$ and $U_{\text {sec }}$ for the interaction between two $1000 \mathrm{~nm}$ silica SPs (solid lines) were also shown.

for CHPs even at this high IS. Aggregates associated with a secondary-minimum could redisperse with a reduction in IS that eliminated the secondary minimum. Increasing the inner radius only slightly increases the values of $U_{\text {pri }}$ and $U_{\max }$ when the IS $\leq 100 \mathrm{mM}$, suggesting similar primary minimum aggregation behaviors for the SPs and CHPs. Conversely, the secondary minimum rapidly decreases with increasing inner radius of the CHPs $(r / R \geq 0.8)$ when the $\mathrm{IS} \leq 100 \mathrm{mM}$. Hence, aggregation of CHPs in a secondary minimum is inhibited with large inner radii. These results indicate great stability of suspension of CHPs with large inner radii at low ISs since the CHPs cannot be aggregated at either primary (due to large energy barriers) or secondary minima, which provides plausible explanation for the monodispersity of CHPs observed in the literature. ${ }^{77-79}$

Figure 8 presents calculated values of $U_{\text {pri }} U_{\text {max }}$ and $U_{\text {sec }}$ for the interaction between two $1000 \mathrm{~nm}$ silica SPs or PHPs with different inner radii under various IS conditions. For the interaction between the two PHPs, the contact points of the inner and outer spheres of the two PHPs face toward or away from each other (see Figure S8, Supporting Information). The former and later interaction orientations were denoted as TWD and AWY, respectively. Figure 8 illustrates that both primary and secondary minima are absent from the IEPs for the interaction between the PHPs in TWD orientation at $r / R \geq$ 0.4. Consequently, these PHPs cannot be aggregated in the TWD orientation. Comparison of Figure 8 with Figure 7 shows that the interaction between the two PHPs in AWY orientation is similar to that between the two CHPs. These results reveal that the PHPs are favored to be aggregated with the contact points facing outward (e.g., Figure S8c, Supporting Information) when the ratio of $r / R$ is above a critical value. The oriented aggregation between HPs has been observed in $\mathrm{He}$ et al. ${ }^{80}$ Such aggregates may have great mobility in the subsurface environments because the interaction energy between an aggregate and a collector surface is mainly determined by those between the surface and the primary particles of the aggregate nearest to the surface ${ }^{45,81}$ and the adhesive forces/ torques between the outermost primary PHPs of the aggregates and the collector surfaces are very weak or even disappear in 
the CPS orientation at large inner radii. The transport of the formed aggregates can be further enhanced by reduced sedimentation as the formed aggregates experience smaller gravitational forces and larger buoyancy forces compared to aggregated SPs. Therefore, the PHP aggregates show great potential for being used in soil remediation due to their much greater abilities to reach contaminated sites compared to SP aggregates.

When SPs aggregate, the resulting clusters can adopt many different forms. Specifically, the aggregation of two SPs will always result in a doublet in the form of a dumbbell. However, a third SP can bond with the doublet in several different ways. ${ }^{82}$ For a particle suspension containing many primary particles, numerous structures may occur for the aggregates due to the randomness of SP bonding. In contrast, the oriented bond of PHPs can significantly reduce the aggregate structures. Accordingly, it would be easier to use PHPs to fabricate aggregates with specific structures. Fabrication of shape controlled aggregates using colloidal materials has spurred great interest due to their attractive morphology-dependent properties. $^{83}$ The formed PHP aggregates may have larger fractal dimensions as their structures (e.g., those in Figure S8c,d of the Supporting Information) are more closed compared to those of SP aggregates. In addition, the rates of PHP aggregation are likely much lower than those of SP aggregation because the probability of the collisions that can result in successful bond is very small between a PHP aggregate with the contact points facing outward and a single PHP or another PHP aggregate. Additional research is warranted to experimentally investigate the kinetics of retention and aggregation of HPs, and to experimentally validate our interaction energy calculations.

It should be noted that our results were obtained by determining DL interaction energies using eq 4. This expression was developed by solving the linearized PoissonBoltzmann (PB) equation and the linearization assumption was thought to be accurate only for $|\psi| \leq 25 \mathrm{mV}$. However, Hogg et al. $^{47}$ (i.e., the developer of eq 4) showed that the region of applicability of the linearized $\mathrm{PB}$ equation is larger than is usually supposed and eq 4 can give fairly accurate results for surface potentials up to $60 \mathrm{mV}$. Indeed, the method of Devereux and de Bruyn ${ }^{84}$ was used to exactly determine DL interaction energies via solving the nonlinearized $\mathrm{PB}$ equation, Figure S10, Supporting Information, shows that the calculated values of $U_{\mathrm{T}}$ for the 1000 and $80 \mathrm{~nm}$ SPs are very similar to those by using eq 4 to determine the $\mathrm{DL}$ energies. The results will also be similar by using the two methods for the HPs since the hollowing only change VDW interaction energies. In addition, the eq 4 was developed based on assuming constant surface potential. If the constant surface charge or the linear superposition approximation approach was adopted, the DL repulsion will be increased, ${ }^{33,85}$ and thus, the HPs will be predicted to have greater mobility. Furthermore, our study considered that the shells of HPs were close-grained. Hallzel and Meireles ${ }^{41}$ showed that the DL repulsion will be increased if the HP shells are coarse-grained and permeable to ions. Therefore, the mobility of the HPs can be further enhanced if the shells of the HPs can be fabricated to be coarse-grained.

\section{CONCLUSIONS}

Through calculating DLVO interaction energies for the CHPs and PHPs, we showed that the retention of the two HPs on a planar surface or within two intercepting half planes via secondary minimum association is inhibited at IS $\leq 100 \mathrm{mM}$ when the shells of the HPs are thin. The retention of the CHPs and PHPs on the collector surface in FPS orientation or within the concave surface in FAV orientation via primary minimum association is similar to the case of SPs at IS $\leq 100 \mathrm{mM}$. However, the CHPs and PHPs with thin shells cannot be retained on the surface in CPS orientation or within the concave site in FTV orientation under these chemical conditions. While the SPs are favorably retained at primary minima at $200 \mathrm{mM}$ due to absence of energy barriers, the retention occurs at secondary minima even at this high IS for the two HPs with thin shells. The orientation dependent energies/forces also occur for the interactions between PHPs, resulting in directional bonds between the PHPs. Our study was the first to examine DLVO interaction energies/forces for HPs and the findings improve our ability to predict and design processes of HP retention and assembly for a variety of environmental and industrial applications such as use of nanomaterials for soil remediation and hierarchical design.

\section{ASSOCIATED CONTENT}

\section{Supporting Information}

The Supporting Information is available free of charge on the ACS Publications website at DOI: 10.1021/acs.langmuir.7b02383.

Methods to calculating interaction force between a HP and two intercepting half planes and interaction energies between two HPs, numerical method of exactly calculating DL energies, zeta potentials of silica and sand, plane view images of a PHP interacting with a planar surface or two intersecting half planes, IEPs for the 1000 and $80 \mathrm{~nm}$ silica SPs, CHPs, PHPs in the CPS orientation, calculated values of $U_{\text {pri }} U_{\text {max }}$ and $U_{\text {sec }}$ for the 1000 and $80 \mathrm{~nm}$ silica PHPs in the FPS orientation, calculated values of $T_{\mathrm{A}} / T_{\mathrm{H}}$ for ZVI PHPs in the CPS orientation, schematic illustration of interaction between two CHPs or between two PHPs and aggregated PHPs. Maps of component of DLVO force along the $y$-direction $\left(F_{y}\right)$ normalized by a hydrodynamic drag torque $\left(F_{\mathrm{D}}\right)$ for interactions between a $1000 \mathrm{~nm}$ silica $\mathrm{SP}$ and two intersecting half planes with an angle of $120^{\circ}$ on the $x-y$ plane at $z=0$, IEPs obtained by calculating DL energies using the analytical equation and numerical methods (PDF).

\section{AUTHOR INFORMATION}

\section{Corresponding Author}

*Phone: +86 1062732850. Fax: +86 1062733596. E-mail: libg@cau.edu.cn.

ORCID *

Chongyang Shen: 0000-0002-2517-3472

Scott Bradford: 0000-0002-3260-2968

\section{Notes}

The authors declare no competing financial interest.

\section{ACKNOWLEDGMENTS}

We acknowledge the financial support provided by the National Natural Science Foundation of China (41271009, 41671222), Beijing Nova Program (Z161100004916116), The National Key Research and Development Program of China 
(2017YFD0800300), and Chinese Universities Scientific Fund (No. 2017ZH001).

\section{REFERENCES}

(1) Kim, S. - W.; Kim, M.; Lee, W. Y.; Hyeon, T. Fabrication of hollow palladium spheres and their successful application to the recyclable heterogeneous catalyst for Suzuki coupling reactions. $\underline{\text {. Am. }}$. Chem. Soc. 2002, 124, 7642-7643.

(2) Wu, S. - H.; Mou, C. - Y.; Lin, H. - P. Synthesis of mesoporous silica nanoparticles. Chem. Soc. Rev. 2013, 42, 3862-3875.

(3) Caruso, F.; Caruso, R. A.; Mohwald, H. Nanoengineering of inorganic and hybrid hollow spheres by colloidal templating. Science 1998, 282, 1111-1114.

(4) Cheng, S.; Yan, D.; Chen, J. T.; Zhuo, R. F.; Feng, J. J.; Li, H. J.; Feng, H. T.; Yan, P. X. Soft-template synthesis and characterization of $\mathrm{ZnO}_{2}$ and $\mathrm{ZnO}$ hollow spheres. I. Phvs. Chem. C 2009, 113, 1363013635 .

(5) Hu, J.; Chen, M.; Fang, X.; Wu, L. Fabrication and application of inorganic hollow spheres. Chem. Soc. Rev. 2011, 40, 5472-5491.

(6) Wang, X.; Feng, J.; Bai, Y.; Zhang, Q.; Yin, Y. Synthesis, properties and applications of hollow micro-/nanostructures. Chem. Rev. 2016, 116, 10983-11060.

(7) Li, S.; Pasc, A.; Fierro, V.; Celzard, A. Hollow carbon spheres, synthesis and applications- a review. I. Mater. Chem. A 2016, 4, 12686-12713.

(8) Gao, C.; Zhang, W.; Li, H.; Lang, L.; Xu, Z. Controllable fabrication of mesoporous $\mathrm{MgO}$ with various morphologies and their adsorption performance for toxic pollutants in water. Cryst. Growth Des. 2008, 8, 3785-3790.

(9) Wang, Y.; Wang, G.; Wang, H.; Liang, C.; Cai, W.; Zhang, L. Chemical-template synthesis of micro/nanoscale magnesium silicate hollow spheres for waste-water treatment. Chem. - Eur. J. 2010, 16, 3497-3503.

(10) Ryan, J. N.; Elimelech, M. Colloid mobilization and transport in groundwater. Colloids Surf. A 1996, 107, 1-56.

(11) Kretzschmar, R; Borkovec, M.; Grolimund, G.; Elimelech, M. Mobile subsurface colloids and their role in contaminant transport. Adv. Agron. 1999, 66, 121-193.

(12) de Jonge, L. W.; Kjaergaard, C.; Moldrup, P. Colloids and colloid-faciliated transport of contaminants in soils: An introduction. Vadose Zone I. 2004, 3, 321-325.

(13) Sen, T. K.; Khilar, K. C. Review on subsurface colloids and colloid-associated contaminant transport in saturated porous media. Adv. Colloid Interface Sci. 2006, 119, 71-96.

(14) McDowell-Boyer, L. M.; Hunt, J. R.; Sitar, N. Particle transport through porous media. Water Resour. Res. 1986, 22, 1901-1921.

(15) Bradford, S. A.; Simunek, J.; Bettahar, M.; van Genuchten, M. T.; Yates, S. R. Significance of straining in colloid deposition: Evidence and implications. Water Resour. Res. 2006, 42, W12S15.

(16) Bradford, S. A.; Torkzaban, S.; Shapiro, A. A theoretical analysis of colloid attachment and straining in chemically heterogeneous porous media. Langmuir 2013, 29, 6944-6952.

(17) Sang, W.; Morales, V. L.; Zhang, W.; Stoof, C. R.; Gao, B.; Schatz, A. L.; Zhang, Y.; Steenhuis, T. S. Quantification of colloid retention and release by straining and energy minima in variably saturated porous media. Environ. Sci. Technol. 2013, 47, 8256-8264.

(18) Molnar, I. L.; Johnson, W. P.; Gerhard, J. I.; Willson, C. S.; O'Carroll, D. M. Predicting colloid transport through saturated porous media: A critical review. Water Resour. Res. 2015, 51, 6804-6845.

(19) Elimelech, M.; O'Melia, C. R. Kinetics of deposition of colloidal particles in porous media. Environ. Sci. Technol. 1990, 24, 1528-1536.

(20) Elimelech, M.; O'Melia, C. R. Effect of particle size on collision efficiency in the deposition of Brownian particles with electrostatic energy barriers. Langmuir 1990, 6, 1153-1163.

(21) Cushing, R. S.; Lawler, D. F. Depth filtration: Fundamental investigation through three-dimensional trajectory analysis. Environ. Sci. Technol. 1998, 32, 3793-3801.

(22) Burdick, G. M.; Bernan, N. S.; Beaudoin, S. P. Hydrodynamic particle removal from surfaces. Thin Solid Films 2005, 488, 116-123.
(23) Bergendahl, J.; Grasso, D. Prediction of colloid detachment in model porous media: Hydrodynamics. Chem. Eng. Sci. 2000, 55, $1523-1532$

(24) Bergendahl, J. A.; Grasso, D. Mechanistic basis for particle detachment from granular media. Environ. Sci. Technol. 2003, 37, $2317-2322$

(25) Torkzaban, S.; Bradford, S. A. Critical role of surface roughness on colloid retention and release in porous media. Water Res. 2016, 88, 274-284.

(26) Li, X.; Zhang, P.; Lin, C. L.; Johnson, W. P. Role of hydrodynamic drag on microsphere deposition and re-entrainment in porous media under unfavorable conditions. Environ. Sci. Technol. 2005, 39, 4012-4020.

(27) Shen, C.; Li, B.; Huang, Y.; Jin, Y. Kinetics of coupled primaryand secondary-minimum depsoition of colloids under unfavorable chemical conditions. Environ. Sci. Technol. 2007, 41, 6976-6982.

(28) Shen, C.; Huang, Y.; Li, B.; Jin, Y. Predicting attachment efficiency of colloid deposition under unfavorable attachment conditions. Water Resour. Res. 2010, 46, W11526.

(29) Bradford, S. A.; Torkzaban, S.; Wiegmann, A. Pore-scale simulations to determine the applied hydrodynamic torque and colloid immobilization. Vadose Zone I. 2011, 10, 252-261.

(30) Bradford, S. A.; Yates, S. R.; Bettahar, M.; Simunek, J. Physical factors affecting the transport and fate of colloids in saturated porous media. Water Resour. Res. 2002, 38, 1327.

(31) Li, X.; Lin, C. L.; Miller, J. D.; Johnson, W. P. Role of grain-tograin contacts on profiles of retained colloids in porous media in the presence of an energy barrier to deposition. Environ. Sci. Technol. 2006, 40, 3769-3774.

(32) Li, X.; Lin, C. L.; Miller, J. D.; Johnson, W. P. Pore-scale observation of microsphere deposition at grain-to-grain contacts over assemblage-scale porous media domains using X-ray microtomography. Environ. Sci. Technol. 2006, 40, 3762-3768.

(33) Verwey, E. J. W.; Overbeek, J. T. G. Theory of the Stability of Lyophobic Colloids; Elsevier: New York, 1948.

(34) Bhattacharjee, S.; Elimelech, M. Surface element integration: A novel technique for evaluation of DLVO interaction between a particle and a flat plate. I. Colloid Interface Sci. 1997, 193, 273-285.

(35) Bhattacharjee, S.; Ko, C. - H.; Elimelech, M. DLVO interaction between rough surfaces. Langmuir 1998, 14, 3365-3375.

(36) Shen, C.; Wang, F.; Li, B.; Jin, Y.; Wang, L. - P.; Huang, Y. Application of DLVO energy map to evaluate interactions between spherical colloids and rough surfaces. Lanomuir 2012, 28, 1468114692.

(37) Boon, N.; Guerrero-Garca, G. I.; van Roji, R.; de la Cruz, M. O. Effective charges and virial pressure of concentrated macroion solutions. Proc. Natl. Acad. Sci. U. S. A. 2015, 112, 9242-9246.

(38) Vogel, N.; Retsch, M.; Fustin, C. - A.; del Campo, A.; Jonas, U. Advances in colloid assembly: The design of structure and hierarchy in two and three dimensions. Chem. Rev. 2015, 115, 6265-6311.

(39) Suresh, L.; Walz, J. Y. Effect of surface roughness on the interaction energy between a colloidal sphere and a flat plate. $\underline{\text {. Colloid }}$ Interface Sci. 1996, 183, 199-213.

(40) Martines, E.; Csaderova, L.; Morgan, H.; Curtis, A. S. G.; Riehle, M. O. DLVO interaction energy between a sphere and a nanopatterned plate. Colloids Surf. A 2008, 318, 45-52.

(41) Mille, M.; Vanderkooi, G. Electrochemical properties of spherical polyelectrolytes: ii. Hollow sphere model for membranous vesicles. I. Colloid Interface Sci. 1977, 61, 455-474.

(42) Angelescu, D. G.; Caragheorgheopol, D. Influence of the shell thickness and charge distribution on the effective interaction between two like-charged hollow spheres. I. Chem. Phvs. 2015, 143, 144902.

(43) Hallez, Y.; Meireles, M. Modeling the electrostatics of hollow shell suspensions: Ion distribution, pair interactions, and many-body effects. Langmuir 2016, 32, 10430-10444.

(44) Ruckenstein, E.; Prieve, D. C. Adsorption and desorption of particles and their chromatographic separation. AIChE J. 1976, 22, 276-283. 
(45) Shen, C.; Wu, L.; Zhang, S.; Ye, H.; Li, B.; Huang, Y. Heteroaggregation of microparticles with nanoparticles changes the chemical reversibility of the microparticles' attachment to planar surfaces. I. Colloid Interface Sci. 2014, 421, 103-113.

(46) Hamaker, H. C. The London-van der Waals attraction between spherical particles. Phvsica 1937, 4, 1058-1072.

(47) Hogg, R. I.; Healy, T. W.; Fuerstenau, D. W. Mutual coagulation of colloidal dispersions. Trans. Faradav Soc. 1966, 62, 1638-1651.

(48) Oliveira, R. Understanding adhesion: a means for preventing fouling. Exp. Therm. Fluid Sci. 1997, 14, 316-322.

(49) Hoek, E. M. V.; Agarwal, G. K. Extended DLVO interactions between spherical particles and rough surfaces. L. Colloid Interface Sci. 2006, 298, 50-58.

(50) Wu, L.; Gao, B.; Tian, Y.; Munoz-Carpena, R.; Zigler, K. J. DLVO interactions of carbon nanotubes with isotropic planar surfaces. Langmuir 2013, 29, 3976-3988.

(51) Pazmino, E.; Trauscht, J.; Dame, B.; Johnson, W. P. Power law size-distributed heterogeneity explains colloid retention on soda lime glass in the presence of energy barrier. Langmuir 2014, 30, 54125421.

(52) Torkzaban, S.; Bradford, S. A.; Walker, S. L. Resolving the coupled effects of hydrodynamics and DLVO forces on colloid attachment in porous media. Langmuir 2007, 23, 9652-9660.

(53) Shang, J.; Flury, M.; Chen, G.; Zhuang, J. Impact of flow rate, water content, and capillary forces on in situ colloid mobilization during infiltration in unsaturated sediments. Water Resour. Res. 2008, 44, W06411.

(54) Sharma, P.; Flury, M.; Zhou, J. Detachment of colloids from a

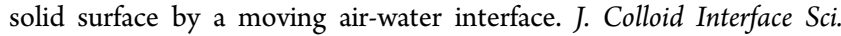
2008, 326, 143-150.

(55) Israelachvili, J. N. Intermolecular and Surface Forces, 2nd ed.; Academic Press: London, 1992.

(56) Wang, C.; Bobba, A. D.; Attinti, R.; Shen, C.; Lazouskaya, V.; Wang, L. - P.; Jin, Y. Retention and transport of silica nanoparticles in saturated porous media: Effect of concentration and particle size. Environ. Sci. Technol. 2012, 46, 7151-7158.

(57) Wang, H.; Huang, Y.; Shen, C.; Wu, J.; Yan, A.; Zhang, H. Cotransport of pesticide acetamiprid and silica nanoparticles in biocharamended sand porous media. L. Environ. Qual. 2016, 45, 1749-1759.

(58) Bergstrom, L. Hamaker constants of inorganic materials. Adv. Colloid Interface Sci. 1997, 70, 125-169.

(59) Bradford, S. A.; Torkzaban, S. Determining parameters and mechanisms of colloid retention and release in porous media. Langmuir 2015, 31, 12096-12105.

(60) Hahn, M. W.; O'Melia, C. R. Deposition and reentrainment of Brownian particles in porou smedia under unfavorable chemical conditions: Some concepts and applications. Environ. Sci. Technol. 2004, 38, 210-220.

(61) Hahn, M. W.; Abadzic, D.; O'Melia, C. R. Aqueous: On the role of secondary minima. Environ. Sci. Technol. 2004, 38, 5915-5924.

(62) Redman, J. A.; Walker, S. L.; Elimelech, M. Bacterial adhesion and transport in porous media: Role of the secondary energy minimum. Environ. Sci. Technol. 2004, 38, 1777-1785.

(63) Tufenkji, N.; Elimelech, M. Correlation equation for predicting single-collector efficiency in physicochemical filtration in saturated porous media. Environ. Sci. Technol. 2004, 38, 529-536.

(64) Nelson, K. E.; Ginn, T. R. New collector efficiency equation for colloid filtration in both natural and engineered flow conditions. Water Resour. Res. 2011, 47, W05543.

(65) Shen, C.; Jin, Y.; Li, B.; Zheng, W.; Huang, Y. Facilitated attachment of nanoparticles at primary minima by nanoscale roughness is susceptible to hydrodynamic drag under unfavorable chemical conditions. Sci. Total Environ. 2014, 466-467, 1094-1102.

(66) Rasmuson, A.; Pazmino, E.; Assemi, S.; Johnson, W. P. Contribution of nano- to microscale roughness to heterogeneity: Closing the gap between unfavorable and favorable colloid attachment conditions. Environ. Sci. Technol. 2017, 51, 2151-2160.

(67) Zhang, W. Nanoscale iron particles for environmental remediation: An overview. I. Nanopart. Res. 2003, 5, 323-332.
(68) Yan, W.; Lien, H. - L.; Koel, B. E.; Zhang, W. Iron nanoparticles for environmental clean-up: recent developments and future outlook. Environ. Sci.: Processes Impacts 2013, 15, 63-77.

(69) Zou, Y.; Wang, X.; Khan, A.; Wang, P.; Liu, Y.; Alsaedi, A.; Hayat, T.; Wang, X. Environmental remediation and applciation of nanoscale zero-valent iron and its composites for the removal of heavy metal ions: A review. Environ. Sci. Technol. 2016, 50, 7290-7304.

(70) Zhan, J.; Zheng, T.; Piringer, G.; Day, C.; Mcpherson, G. L.; Lu, Y.; Papadopoulos, K.; John, V. T. Transport characteristics of nanoscale functional zerovalent iron/silica composites for in situ remediation of trichloroethylene. Environ. Sci. Technol. 2008, 42, $8871-8876$

(71) Johnson, R. L.; Nurmi, J. T.; O’Brien Johnson, G. S.; Fan, D.; O’Brien Johnson, R. L.; Shi, Z.; Salter-Blanc, A. J.; Tratnyek, P. G.; Lowry, G. V. Field-scale transport and transformation of carboxymethylcellulose-stabilized nano zero-valent iron. Environ. Sci. Technol. 2013, 47, 1573-1580.

(72) Kocur, C. M.; O'Carroll, D. M.; Sleep, B. E. Impact of nZVI stability on mobility in porous media. I. Contam. Hvdrol. 2013, 145 $17-25$.

(73) Raychoudhury, T.; Tufenkji, N.; Ghoshal, S. Straining of polyelectrolyte-stabilized nanoscale zero valent iron particles during transport through granular porous media. Water Res. 2014, 50, 80-89.

(74) O'Carroll, D.; Sleep, B.; Krol, M.; Boparai, H.; Kocur, C. Nanoscale zero valent iron and bimetallic particles for contaminated site remediation. Adv. Water Resour. 2013, 51, 104-122.

(75) Johnson, W. P.; Li, X.; Yal, G. Colloid retention in porous media: Mechanistic confirmation of wedging and retention in zones of flow stagnation. Environ. Sci. Technol. 2007, 41, 1279-1287.

(76) Li, T.; Jin, Y.; Huang, Y.; Li, B.; Shen, C. Observed dependence of colloid detachment on the concentration of initially attached colloids and collector surface heterogeneity in porous media. Environ. Sci. Technol. 2017, 51, 2811-2820.

(77) Kumar, A.; Gu, S. Modeling impingement of hollow metal droplets onto a flat surface. Int. I. Int. I. Heat Fluid Flow 2012, 37, 189-195.

(78) Wei, W.; Ma, G. - H.; Hu, G.; Yu, D.; Mcleish, T.; Su, Z. - G.; Shen, Z. - Y. Preparation of hierarchical hollow $\mathrm{CaCO}_{3}$ particles and the application as anticancer drug carrier. I. Am. Chem. Soc. 2008, 130, $15808-15810$.

(79) Deng, Z.; Chen, M.; Zhou, S.; You, B.; Wu, L. A novel method for the fabrication of monodisperse hollow silica spheres. Langmuir 2006, 22, 6403-6407.

(80) He, T.; Chen, D.; Jiao, X.; Xu, Y.; Gu, Y. Surfactant-assisted solvothermal synthesis of $\mathrm{Co}_{3} \mathrm{O}_{4}$ hollow spheres with orientedaggregation nanostructures and tunable particle size. Langmuir 2004, 20, 8404-8408

(81) Lin, S.; Wiesner, M. R. Deposition of aggregated nanoparticles-a theoretical and experimental study on the effect of aggregation state on the affinity between nanoparticles and a collector surface. Environ. Sci. Technol. 2012, 46, 13270-13277.

(82) Elimelech, M.; Gregory, J.; Jia, X.; Williams, R. A. Particle Deposition and Aggregation: Measurement, Modeling and Simulation; Butterworth-Helnemann: Oxford, England, 1995.

(83) $\mathrm{Xu}, \mathrm{H}$.; Wang, W.; Zhou, L. A growth model of single crystalline hollow spheres: Oriented attachment of $\mathrm{Cu}_{2} \mathrm{O}$ nanoparticles to the single crystalline shell wall. Crust. Growth Des. 2008, 8, 3486-3489.

(84) Devereux, O. F.; de Bruyn, P. L. Interaction of Plane Parallel Double Layers; MIT Press: Cambridge, 1963.

(85) Adamczyk, Z.; Weronski, P. Application of the DLVO theory for particle deposition problems. Adv. Colloid Interface Sci. 1999, 83, 137226. 


\section{Supporting Information}

DLVO Interaction Energies between Hollow Spherical Particles and Collector Surfaces

Chongyang Shen, ${ }^{\dagger}$ Scott Bradford, ${ }^{\ddagger}$ Zhan Wang, ${ }^{\dagger, \S}$ Yuanfang Huang, ${ }^{\dagger}$ Yulong Zhang, ${ }^{\S}$ and Baoguo $\mathrm{Li}^{\dagger}$,*

'Department of Soil and Water Sciences, China Agricultural University, Beijing 100193, China.

${ }^{\star}$ U.S. Salinity Laboratory USDA, ARS, Riverside, California 92507-4617, United States.

${ }^{\S}$ College of Land and Environment, Shenyang Agricultural University, Shenyang, Liaoning 110866, China.

"Corresponding author: Phone, +86 1062732850; Fax, +86 1062733596; Email, libg@cau.edu.cn

Number of Tables: 1

Number of Figures: 10 


\section{Calculation of interaction force between a HP and two intersecting half planes}

The Cartesian coordinate system was used to determine separation distances between a HP (i.e., CHP or PHP) and two half planes in Figure 1b and Figure S1e and S1f of Supporting Information. The $z$ axis of the coordinate system coincides with the line where the two half planar surfaces intersect, and the $y-z$ plane bisects the two intersecting half planar surfaces. The separation distances between the HP and the two half planar surfaces in the coordinate system are shown in Table S2 of Supporting Information. The total DLVO interaction forces along $x$-direction $\left(F_{\mathrm{x}}\right)$ and $y$-direction $\left(F_{\mathrm{y}}\right)$ for the interaction configuration in Figure 1 are calculated as $F_{1} \cos \left(\frac{\alpha}{2}\right)-F_{2} \cos \left(\frac{\alpha}{2}\right)$ and $F_{1} \sin \left(\frac{\alpha}{2}\right)+F_{2} \sin \left(\frac{\alpha}{2}\right)$, respectively. $F_{1}$ and $F_{2}$ are the interaction forces between the HP and the left and right half planar surface, respectively, and $\alpha$ is the angle between the two intersecting half planar surfaces. The force between the HP and a half planar surface is the derivative of the interaction energy between the HP and the half planar surface with respect to separation distance. Details about calculation of the interaction energy between the HP and half planar surface using surface element integration technique have been given in the section of Theory. A Matlab program was developed to implement the calculations in three-dimensional space. 


\section{Calculation of interaction energies between two HPs}

The total interaction energy $\left(U_{\mathrm{T}}\right)$ between two identical CHPs in Figure S8(a) of Supporting Information is calculated by

$$
U_{\mathrm{T}}(H)=U_{\mathrm{T}}^{\mathrm{AA}}(H)-2 U_{\mathrm{VDW}}^{\mathrm{Aa}}(H+R-r)-U_{\mathrm{VDW}}^{\mathrm{aa}}(H+2 R-2 r)
$$

where $H$ is separation distance between the two CHPs, $R$ and $r$ are the outer and inner radii of the CHPs, respectively, $U_{\mathrm{T}}^{\mathrm{AA}}$ is the total interaction energy between outer spheres of the two CHPs, $U_{\mathrm{VDw}}^{\mathrm{Aa}}$ is the VDW interaction energy between the outer sphere of a CHP and the inner sphere of the other CHP, $U_{\text {VDW }}^{\text {aa }}$ is the VDW interaction energy between inner spheres of the two CHPs. The total energy for the interaction between two identical PHPs with the contact points facing each other in Figure S8(b1) of Supporting Information is calculated by

$$
U_{\mathrm{T}}(H)=U_{\mathrm{T}}^{\mathrm{AA}}(H)-2 U_{\mathrm{VDW}}^{\mathrm{Aa}}(H)-U_{\mathrm{VDW}}^{\mathrm{aa}}(H)
$$

The total energy for the interaction between two identical PHPs with the contact points facing away from each other in Figure S8(b2) of Supporting Information is calculated by

$$
U_{\mathrm{T}}(H)=U_{\mathrm{T}}^{\mathrm{AA}}(H)-2 U_{\mathrm{VDW}}^{\mathrm{Aa}}(H+2 R-2 r)-U_{\mathrm{VDW}}^{\mathrm{aa}}(H+4 R-4 r)
$$

The surface element integration technique was used to calculate the VDW, DL, and BR energies between two spheres and details about the calculations can be referred to Bhattacharjee et al. ${ }^{1}$ and Shen et al. ${ }^{2}$ 


\section{Exact evaluation of differential DL interaction energies between two planar surfaces}

The method of Devereux and de Bruyn ${ }^{3}$ was used to exactly calculate DL energies between two planar surfaces per unit area $E_{\mathrm{DL}}$ by solving the one-dimensional nonlinear Poisson-Boltzmann (PB) equation. Briefly, the nonlinear PB equation is written as

$$
\frac{d^{2} \Psi}{d \xi^{2}}=\sinh \Psi
$$

where $\xi(=\kappa x)$ is dimensionless distance, $\Psi$ is dimensionless potential, given by

$$
\Psi=\frac{v e \psi}{k T}
$$

where $v$ is valence of ions in the electrolyte, $e$ is charge of a proton. Equation (S4) can be solved by using the following boundary conditions:

$$
\left.\begin{array}{ll}
\Psi=\Psi_{\mathrm{p}} & \text { at } \xi=0 \\
\Psi=\Psi_{\mathrm{d}} & \text { at } \xi=\xi_{\mathrm{H}}
\end{array}\right\}
$$

which correspond to the conditions:

$$
\left.\begin{array}{ll}
\psi=\psi_{\mathrm{p}} & \text { at } x=0 \\
\psi=\psi_{\mathrm{d}} & \text { at } x=H
\end{array}\right\}
$$

By integrating Equation (S4) once, the following equation can be obtained:

$$
\frac{\mathrm{d} \Psi}{\mathrm{d} \xi}= \pm \sqrt{2 \cosh \Psi+C}
$$

where $C$ is an integration constant.

If both interacting planar surfaces are positively charged and the value of $\psi_{\mathrm{p}}$ is greater than $\psi_{\mathrm{d}}$, the following expressions which describe the dimensional distance as a function of dimensionless potential can be obtained from Equation (S8) for three ranges of $C$ :

$$
\begin{aligned}
\xi= & 2 \exp \left(-\frac{\Psi_{\text {min }}}{2}\right)\left\{F\left[\exp \left(-\Psi_{\min }\right), \sin ^{-1}\left(\sqrt{\exp \left(\Psi_{\min }-\Psi\right)}\right)\right]\right. \\
& \left.-F\left[\exp \left(-\Psi_{\text {min }}\right), \sin ^{-1}\left(\sqrt{\exp \left(\Psi_{\text {min }}-\Psi_{\mathrm{p}}\right.}\right)\right]\right\}
\end{aligned} \text { at } C \leq-2 \text { and } \frac{\mathrm{d} \Psi}{\mathrm{d} \xi}<0
$$




$$
\begin{gathered}
\xi=2 \exp \left(-\frac{\Psi_{\text {min }}}{2}\right)\left\{2 F\left[\exp \left(-\Psi_{\min }\right), \frac{\pi}{2}\right]-F\left[\exp \left(-\Psi_{\min }\right), \sin ^{-1}\left(\sqrt{\exp \left(\Psi_{\min }-\Psi_{\mathrm{p}}\right)}\right)\right] \text { at } C \leq-2 \text { and } \frac{\mathrm{d} \Psi}{\mathrm{d} \xi}\right. \\
\left.-F\left[\exp \left(-\Psi_{\min }\right), \sin ^{-1}\left(\sqrt{\exp \left(\Psi_{\min }-\Psi\right)}\right)\right]\right\} \\
\xi=F\left[\sqrt{\frac{2-C}{4}}, \pi-2 \tan ^{-1} \sqrt{\exp (\Psi)}\right]-F\left[\sqrt{\frac{2-C}{4}}, \pi-2 \tan ^{-1} \sqrt{\exp \left(\Psi_{\mathrm{p}}\right)}\right] \text { at }-2 \leq C \leq 2 \\
\xi=\frac{2}{b}\left\{F\left[\sqrt{1-\frac{1}{b^{4}}}, \tan ^{-1} \operatorname{bexp}\left(\frac{\Psi_{\mathrm{p}}}{2}\right)\right]-F\left[\sqrt{1-\frac{1}{b^{4}}}, \tan ^{-1} \operatorname{bexp}\left(\frac{\Psi}{2}\right)\right]\right\} \text { at } 2 \leq C \quad \text { (S12) }
\end{gathered}
$$

where $F$ represents the elliptic integral of the first kind, $\Psi_{\min }$ is the minimum

dimensionless potential between the two interacting surfaces for a given value of $H$ in Equation (S9) or an imagined minimum dimensionless potential beyond the interacting surfaces $^{3}$ in Equation (S10). $\Psi_{\min }$ and $b$ are related to $C$ by the following expressions:

$$
\begin{gathered}
C=-2 \cosh \left(\Psi_{\min }\right) \\
b=\sqrt{\frac{C}{2}+\frac{1}{2} \sqrt{C^{2}-4}}
\end{gathered}
$$

Substituting $\xi_{\mathrm{H}}$ and $\Psi_{\mathrm{d}}$ into Equation (S9)-(S12), the value of $C$ (or $\Psi_{\min }$ and $b$ ) can be obtained through iteration. Note that in order to decide which equation to use to obtain $C$ for a given $H$, the separation distance $H_{1}$ (or $\xi_{H_{1}},=\kappa H_{1}$ ) corresponding to $C=2$ and the separation distance $H_{2}$ (or $\xi_{H_{2}},=\kappa H_{2}$ ) corresponding to $C=-2$ have to be determined firstly. The expressions used to determine $\xi_{H_{1}}$ and $\xi_{H_{2}}$ are in the following

$$
\begin{gathered}
\xi_{H_{1}}=F\left[0, \pi-2 \tan ^{-1} \sqrt{\exp \left(\frac{\Psi_{\mathrm{d}}}{2}\right)}\right]-F\left[0, \pi-2 \tan ^{-1} \sqrt{\exp \left(\frac{\Psi_{\mathrm{p}}}{2}\right)}\right] \\
\xi_{H_{2}}=\ln \left\{\frac{\left[1-\exp \left(-\Psi_{\mathrm{p}} / 2\right)\right]\left[1+\exp \left(-\Psi_{\mathrm{d}} / 2\right)\right]}{\left[1-\exp \left(-\Psi_{\mathrm{d}} / 2\right)\right]\left[1+\exp \left(-\Psi_{\mathrm{p}} / 2\right)\right]}\right\}
\end{gathered}
$$

Equation (S11) and (S12) and are used for $\xi_{H_{1}} \leq \xi \leq \xi_{H_{2}}$ and $\xi \leq \xi_{H_{1}}$, respectively. To determine which equation [i.e., Equation (S9) or (S10)] to use to calculate $\Psi_{\min }$ at $C \leq-2$, an additional expression has to be adopted to determine $\xi_{\min }$ (i.e., the dimensionless distance 
at which the potential reaches minimum), given by

$$
\left.\xi_{\min }=2 \exp \left(-\frac{\Psi_{d}}{2}\right)\left\{F\left[\exp \left(-\Psi_{\mathrm{d}}\right), \frac{\pi}{2}\right]-F\left[\exp \left(-\Psi_{\mathrm{d}}\right), \sin ^{-1} \sqrt{\exp \left(\Psi_{\mathrm{d}}-\Psi_{\mathrm{p}}\right)}\right)\right]\right\}
$$

Equation (S9) and (S10) are used to calculate $\Psi_{\min }$ or $C$ at $C \leq-2$ for $\xi \leq \xi_{\min }$ and $\xi \geq \xi_{\min }$, respectively.

If $\Psi_{\min }$ was obtained using Equation (10) at $C \leq-2$, the value of $E_{\mathrm{DL}}$ can then be calculated using the following expression for $C \leq-2$ :

$$
\begin{aligned}
E_{\mathrm{DL}} & =-\frac{2 n k T}{\kappa}\left[\frac{\xi_{\mathrm{H}}}{2}\left[3 \exp \Psi_{\text {min }}-2-\exp \left(-\Psi_{\min }\right)\right]+2 \sqrt{2 \cosh \Psi_{\mathrm{p}}-2 \cosh \Psi_{\text {min }}}\right. \\
& -2 \sqrt{2 \cosh \Psi_{\mathrm{d}}-2 \cosh \Psi_{\text {min }}}+4 \exp \left(\frac{\Psi_{\text {min }}}{2}\right)\left(E\left\{\exp \left(-\Psi_{\min }\right), \sin ^{-1}\left[\exp \left(\frac{\Psi_{\text {min }}-\Psi_{\mathrm{p}}}{2}\right)\right]\right\}\right. \\
& \left.\left.+E\left\{\exp \left(-\Psi_{\text {min }}\right), \sin ^{-1}\left[\exp \left(\frac{\Psi_{\text {min }}-\Psi_{\mathrm{d}}}{2}\right)\right]\right\}-2 E\left[\exp \left(-\Psi_{\text {min }}\right), \frac{\pi}{2}\right]\right)\right]-E_{\mathrm{DL}}^{\infty}
\end{aligned}
$$

where $E$ represents the elliptic integral of the second kind, $E_{\mathrm{DL}}^{\infty}$ is the differential interaction energy when the separation distance is infinite ${ }^{3}$, which is given by

$$
E_{\mathrm{DL}}=-\frac{2 n k T}{\kappa}\left[8-4 \cosh \left(\frac{\Psi_{\mathrm{p}}}{2}\right)-4 \cosh \left(\frac{\Psi_{\mathrm{d}}}{2}\right)\right]
$$

If $\Psi_{\min }$ was obtained using Equation (9) (i.e., when $\Psi_{\min }$ is located beyond the interacting surfaces), the following expression has to be used to calculate $E_{\mathrm{DL}}$ :

$$
\begin{aligned}
E_{\mathrm{DL}}= & -\frac{2 n k T}{\kappa}\left[\frac{\xi_{\mathrm{H}}}{2}[3 \exp (p)-2-\exp (-p)]+2 \sqrt{2 \cosh \Psi_{\mathrm{p}}-2 \cosh (p)}\right. \\
& -2 \sqrt{2 \cosh \Psi_{\mathrm{d}}-2 \cosh (p)}+4 \exp \left(\frac{p}{2}\right)\left(E\left\{\exp (-p) \sin ^{-1}\left[\exp \left(\frac{p-\Psi_{\mathrm{p}}}{2}\right)\right]\right\}\right. \\
& \left.\left.-E\left\{\exp (-p), \sin ^{-1}\left[\exp \left(\frac{p-\Psi_{\mathrm{d}}}{2}\right)\right]\right\}\right)\right]-E_{\mathrm{DL}}^{\infty}
\end{aligned}
$$

where $p=\cosh ^{-1}\left(-\frac{C}{2}\right)$.

By using Equation (11) and (12) to calculate $C$, the following expressions can then be 
used to calculate $E_{\mathrm{DL}}$ at $-2 \leq C \leq 2$ and $C \geq 2$ :

$$
\begin{aligned}
& E_{\mathrm{DL}}=-\frac{2 n k T}{\kappa}\left(\xi_{\mathrm{H}}\left(-\frac{C}{2}-1\right)-2 \sqrt{2} \sqrt{\frac{\left(\cosh \Psi_{\mathrm{d}}-1\right)\left(\cosh \Psi_{\mathrm{d}}+C / 2\right)}{\cosh \Psi_{\mathrm{d}}+1}}\right. \\
& -2 \sqrt{2} \sqrt{\frac{\left(\cosh \Psi_{\mathrm{p}}-1\right)\left(\cosh \Psi_{\mathrm{p}}+C / 2\right)}{\cosh \Psi_{\mathrm{p}}+1}}+4\left\{2 E\left(\sqrt{\frac{2-C}{4}}, \frac{\pi}{2}\right)\right. \\
& \left.-E\left[\sqrt{\frac{2-C}{4}}, \sin ^{-1}\left(\frac{1}{\cosh \left(\Psi_{\mathrm{p}} / 2\right)}\right)\right]--E\left[\sqrt{\frac{2-C}{4}}, \sin ^{-1}\left(\frac{1}{\cosh \left(\Psi_{\mathrm{d}} / 2\right)}\right)\right]\right\}-E_{\mathrm{DL}}^{\infty} \\
& E_{\mathrm{DL}}=-\frac{2 n k T}{\kappa}\left[\xi_{\mathrm{H}}\left(1-\frac{C}{2}\right)-2 \sqrt{2 \cosh \Psi_{\mathrm{d}}+C}+2 \sqrt{2 \cosh \Psi_{\mathrm{p}}+C}\right. \\
& -4 \exp \left(\frac{\Psi_{\mathrm{p}}}{2}\right) \sqrt{\frac{1 / b^{2}+\exp \Psi_{\mathrm{p}}}{b^{2}+\exp \Psi_{\mathrm{p}}}}+4 \exp \left(\frac{\Psi_{\mathrm{d}}}{2}\right) \sqrt{\frac{1 / b^{2}+\exp \Psi_{\mathrm{d}}}{b^{2}+\exp \Psi_{\mathrm{d}}}} \\
& \left.+4 b\left(E\left\{\sqrt{1-\frac{1}{b^{4}}}, \tan ^{-1}\left[\operatorname{bexp}\left(-\frac{\Psi_{\mathrm{d}}}{2}\right)\right]\right\}-E\left\{\sqrt{1-\frac{1}{b^{4}}}, \tan ^{-1}\left[\operatorname{bexp}\left(-\frac{\Psi_{\mathrm{p}}}{2}\right)\right]\right\}\right)\right]-E_{\mathrm{DL}}^{\infty}
\end{aligned}
$$

It is worthwhile mentioning that the aforementioned method can also be used to calculate $E_{\mathrm{DL}}$ for $\psi_{\mathrm{p}}<\psi_{\mathrm{d}}$ and for two interacting negatively charged surfaces since the energy of a system can depend ultimately on neither the spatial coordinates of the system nor the sign conventions used to describe the system and surface charge $\mathrm{e}^{3}$. 
Table S1. Zeta potentials ( $\mathrm{mV}$ ) of silica and sand in $\mathrm{NaCl}$ solutions with different ISs at pH 7.

\begin{tabular}{|c|c|c|}
\hline IS $(\mathrm{mM})$ & silica HPs & sand \\
\hline 1 & -55.8 & -43.6 \\
\hline 10 & -37.3 & -39.1 \\
\hline 100 & -33.9 & -32.1 \\
\hline 200 & -23.6 & -15.7 \\
\hline
\end{tabular}


Table S2. Separation distances between a HP and two half planes in Figure 1. The center of the HP is assigned the coordinates $(x, y, z), \alpha$ is the angle between the two half planes, $a_{\mathrm{p}}$ is radius of the colloid.

\begin{tabular}{|c|c|}
\hline Separation distance & Equations \\
\hline \multirow{2}{*}{$H_{1}$} & $\sqrt{x^{2}+y^{2}} \cdot \sin \left(\frac{\alpha}{2}-\arccos \left(\frac{y}{\sqrt{x^{2}+y^{2}}}\right)\right)-a_{\mathrm{p}} \quad(x<0)$ \\
\cline { 2 - 3 } & $\sqrt{x^{2}+y^{2}} \cdot \sin \left(\frac{\alpha}{2}+\arccos \left(\frac{y}{\sqrt{x^{2}+y^{2}}}\right)\right)-a_{\mathrm{p}} \quad(x>0)$ \\
\hline \multirow{3}{*}{$H_{2}$} & $\sqrt{x^{2}+y^{2}} \cdot \sin \left(\frac{\alpha}{2}+\arccos \left(\frac{y}{\sqrt{x^{2}+y^{2}}}\right)\right)-a_{\mathrm{p}} \quad(x<0)$ \\
\cline { 2 - 3 } & $\sqrt{x^{2}+y^{2}} \cdot \sin \left(\frac{\alpha}{2}-\arccos \left(\frac{y}{\sqrt{x^{2}+y^{2}}}\right)\right)-a_{\mathrm{p}} \quad(x>0)$ \\
\hline
\end{tabular}



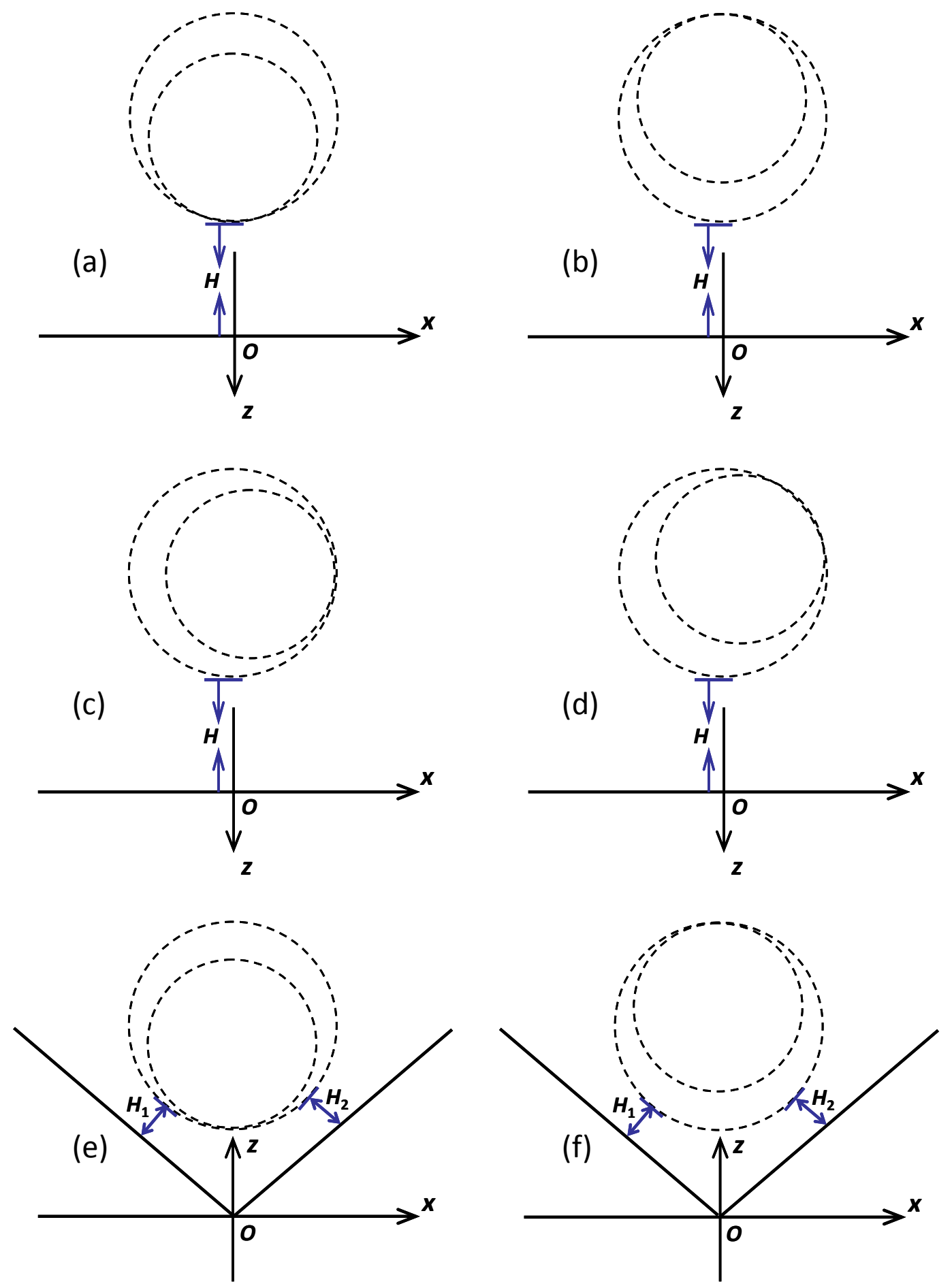

Figure S1. Plan view images of interaction of a PHP with a planar surface or with two intersecting half planes. 

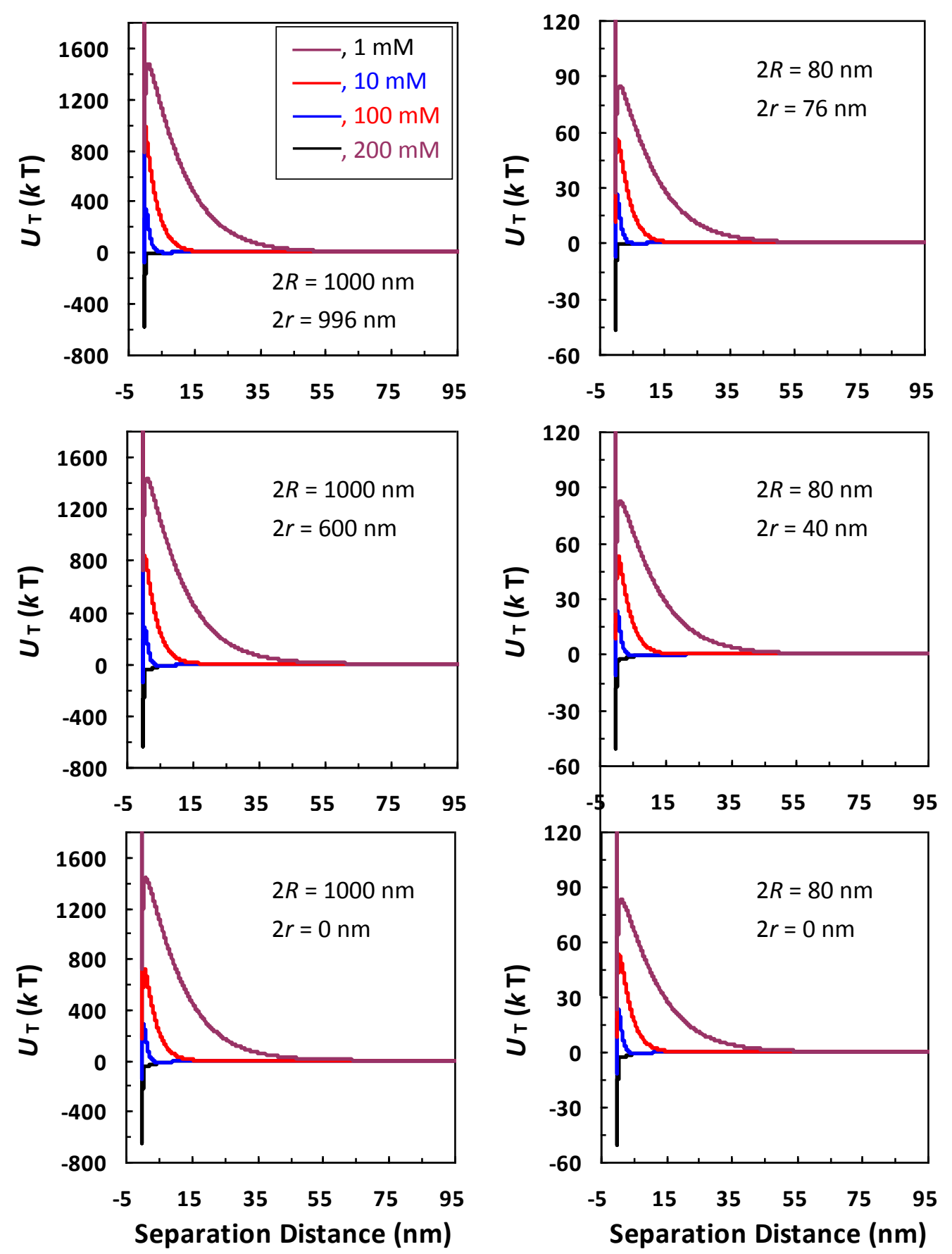

Figure S2. IEPs for the $1000 \mathrm{~nm}$ and $80 \mathrm{~nm}$ SPs and CHPs with different inner radii at different ISs. The IEPs for the 1000 and $80 \mathrm{~nm}$ SPs (i.e., $2 r=0 \mathrm{~nm}$ ) are also shown. 

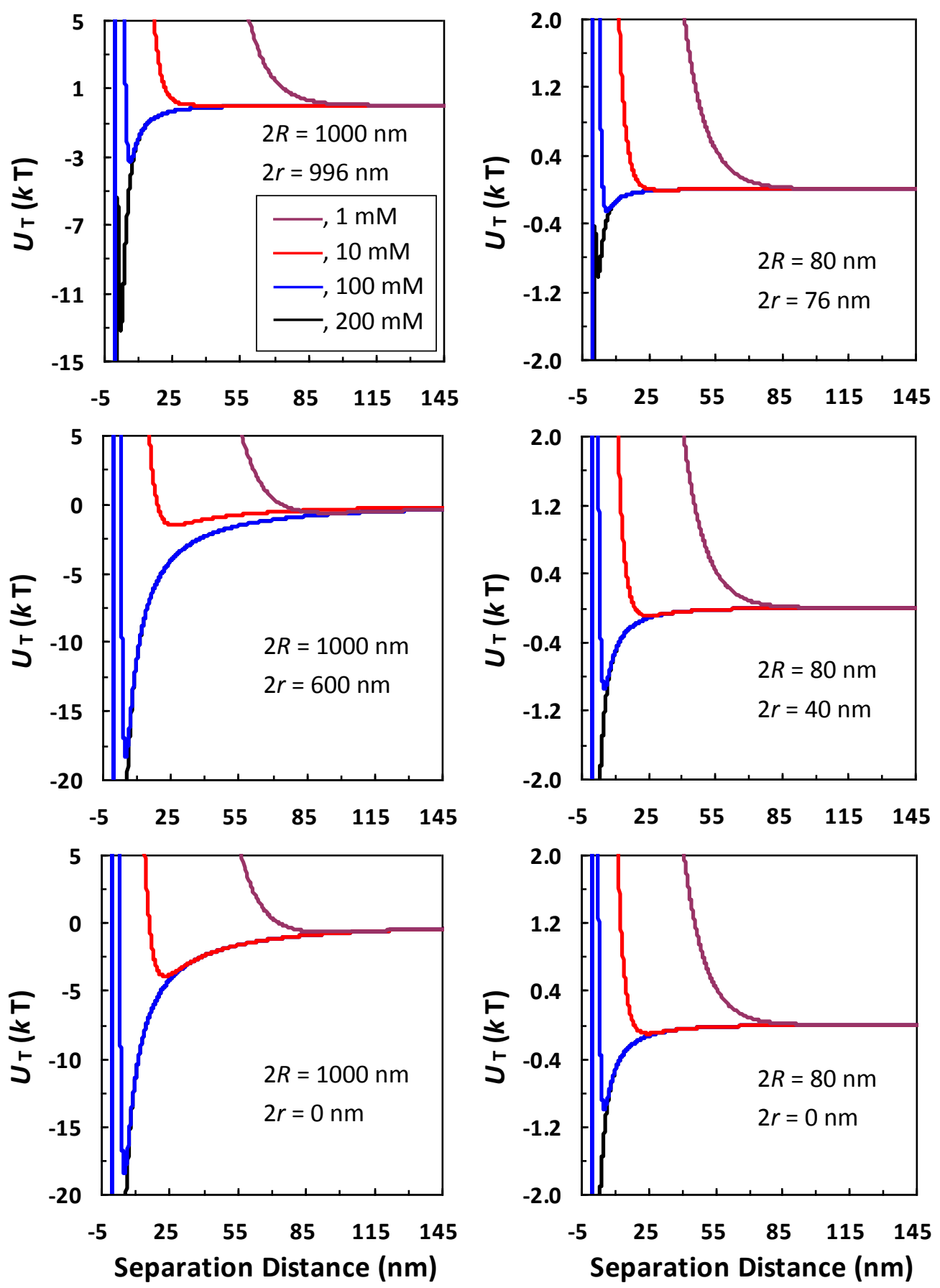

Figure S3. Re-plotted IEPs in Figure S2 of Supporting Information for the $1000 \mathrm{~nm}$ and $80 \mathrm{~nm}$ SPs and CHPs of different inner radii at different ISs to highlight the depth and location of secondary minima. The re-plotted IEPs for the 1000 and $80 \mathrm{~nm}$ SPs (i.e., $r=0$ $\mathrm{nm})$ are also shown. 

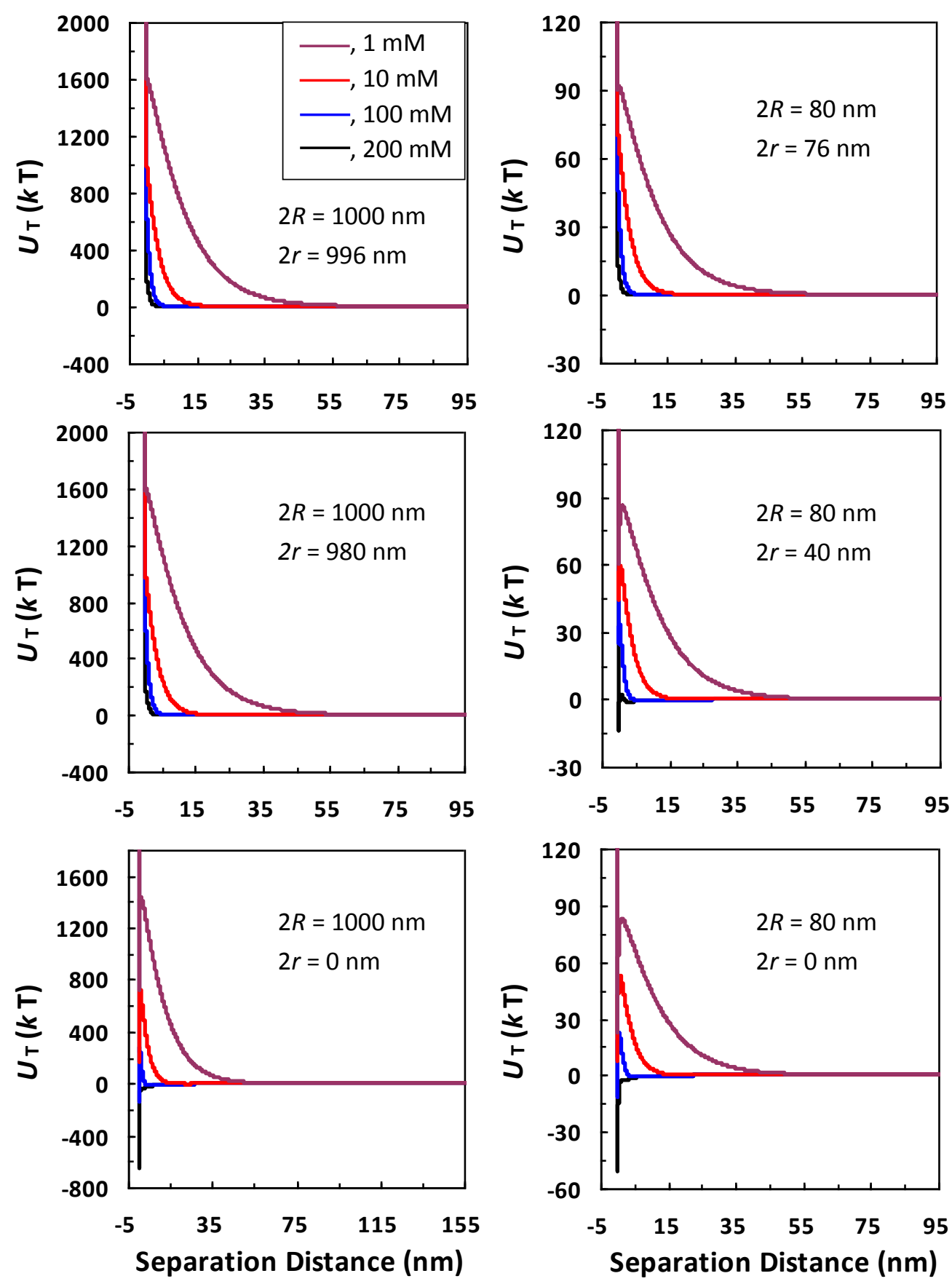

Figure S4. IEPs for the $1000 \mathrm{~nm}$ and $80 \mathrm{~nm}$ silica PHPs with different inner radii in the CPS orientation at different ISs. The IEPs for the 1000 and $80 \mathrm{~nm}$ SPs (i.e., $2 r=0 \mathrm{~nm}$ ) are also shown. 

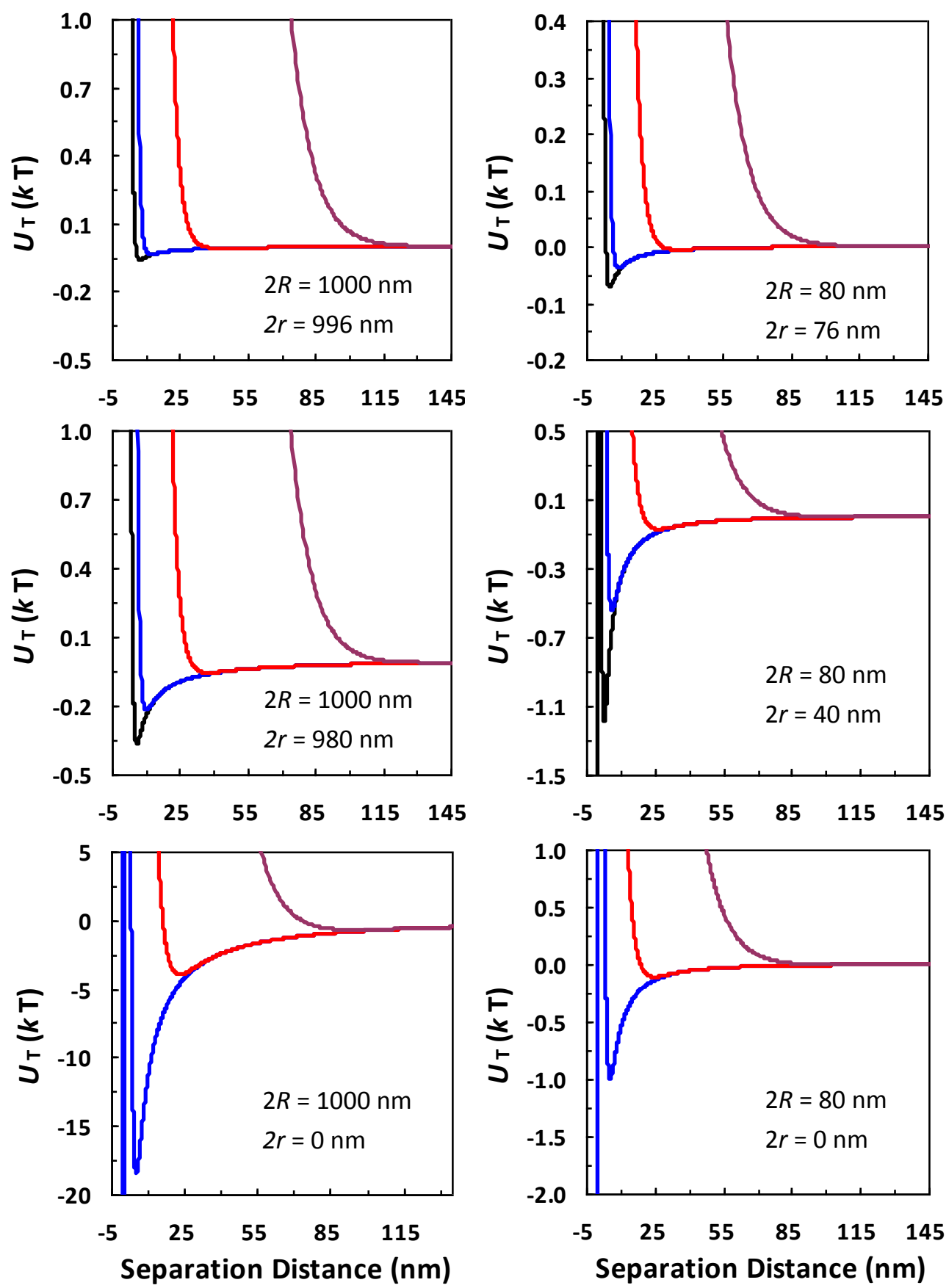

Figure S5. Re-plotted IEPs in Figure S4 for the $1000 \mathrm{~nm}$ and $80 \mathrm{~nm}$ silica PHPs with different inner radii in the CPS orientation at different ISs to highlight the depth and location of secondary minima. The re-plotted IEPs for the 1000 and $80 \mathrm{~nm}$ SPs (i.e., $r=0$ nm) are also shown. 

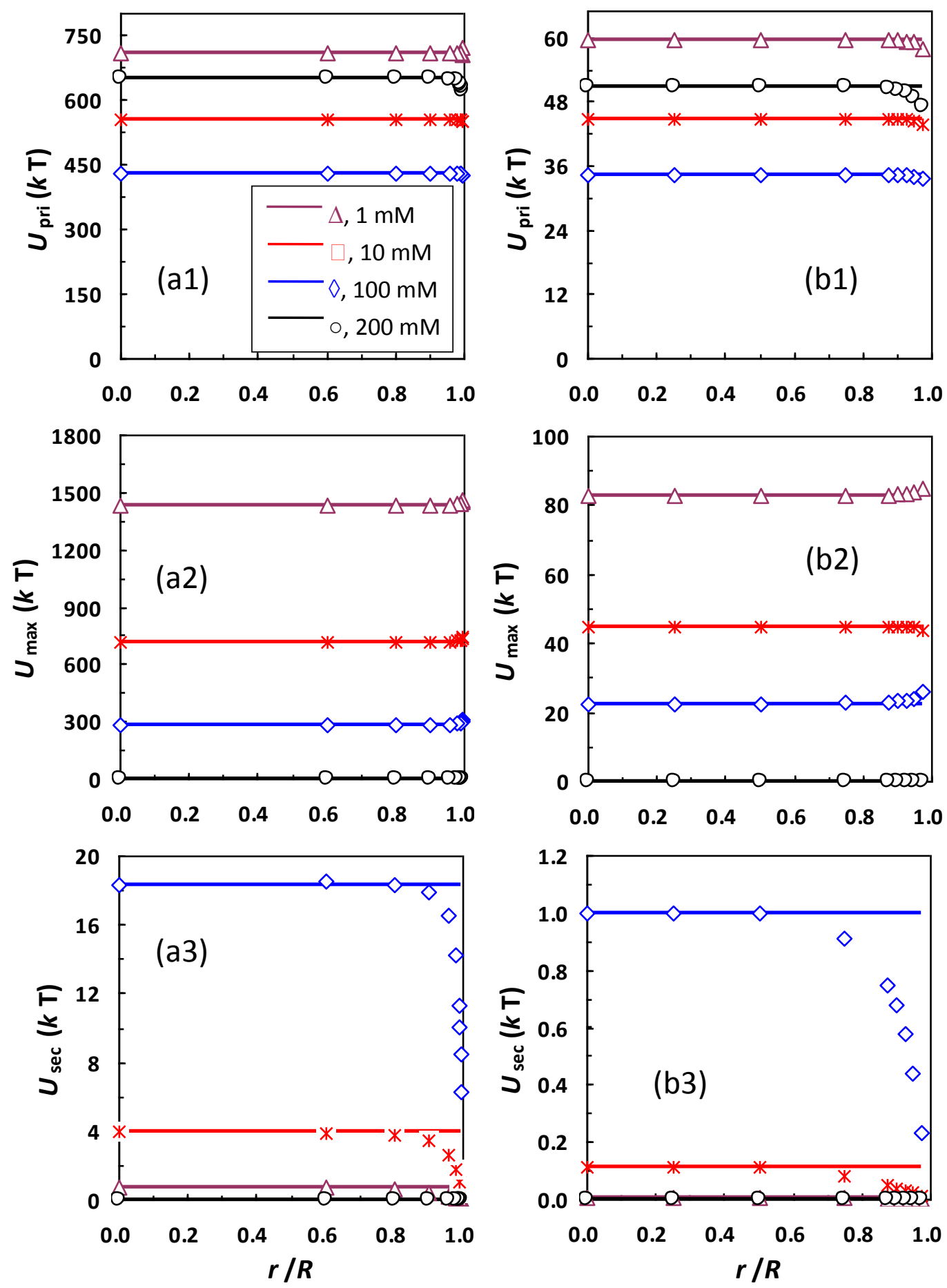

Figure S6. Calculated values of $U_{\mathrm{pri}}, U_{\mathrm{max}}, U_{\mathrm{sec}}$ for the (a) 1000 and (b) $80 \mathrm{~nm}$ silica PHPs with different inner radii in the FPS orientation at different ISs. The values of $U_{\text {pri }}, U_{\max }$, and $U_{\text {sec }}$ for the 1000 and $80 \mathrm{~nm}$ silica SPs (solid lines) were also shown. 

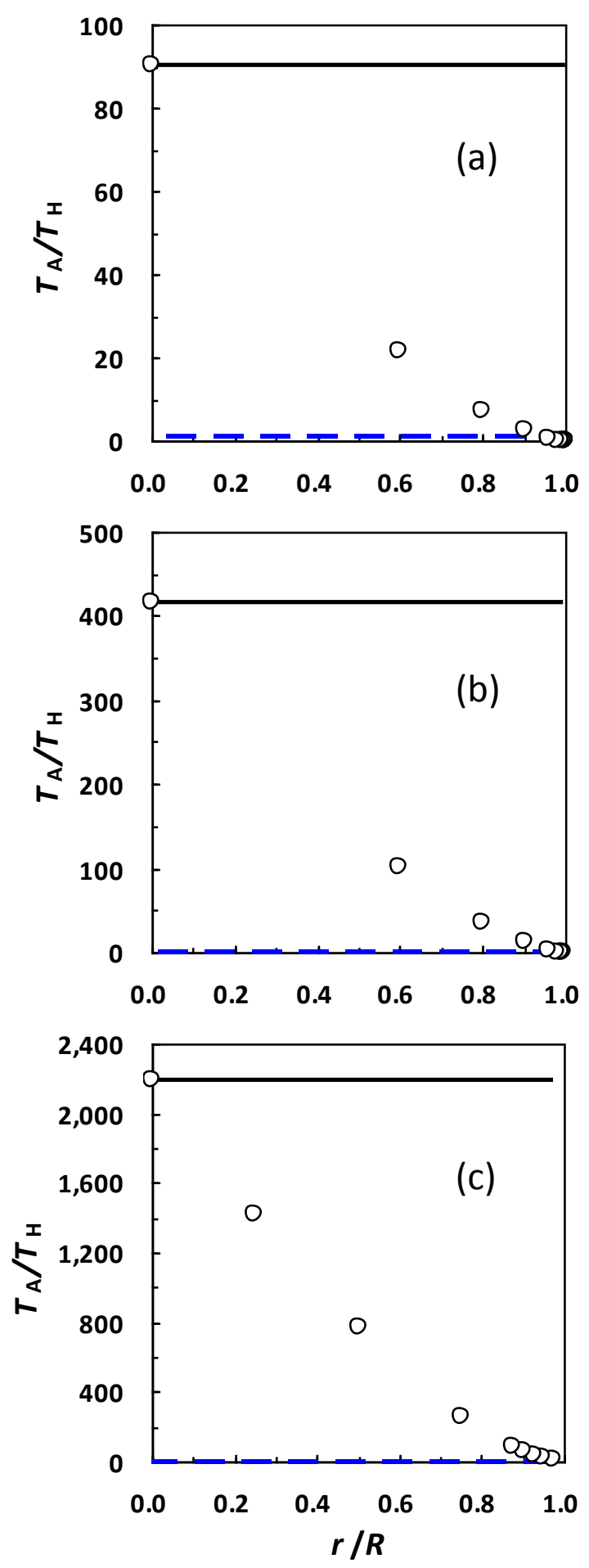

Figure S7. Calculated adhesive torques $\left(T_{\mathrm{A}}\right)$ normalized by a hydrodynamic drag torque ( $T_{\mathrm{H}}$ ) for a (a) 10000, (b) 1000, or (c) $80 \mathrm{~nm}$ ZVI PHP in the CPS orientation with different shell thicknesses. Soil lines represent values of $T_{\mathrm{A}} / T_{\mathrm{H}}$ for ZVI SPs, and dashed line represents $T_{\mathrm{A}} / T_{\mathrm{H}}=1$ with $A_{\mathrm{H}}=1.6 \times 10^{-20} \mathrm{~J}^{4}$ 
(a)

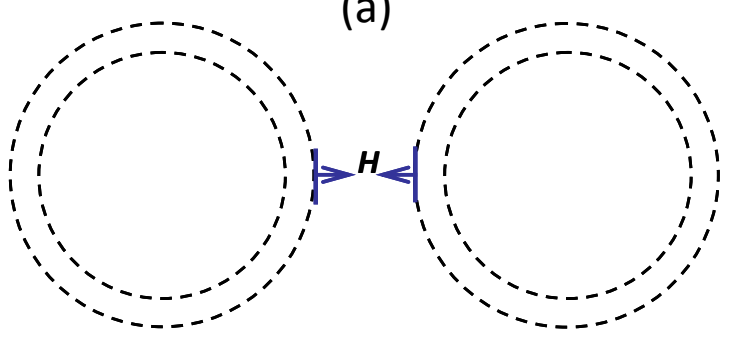

(b1)

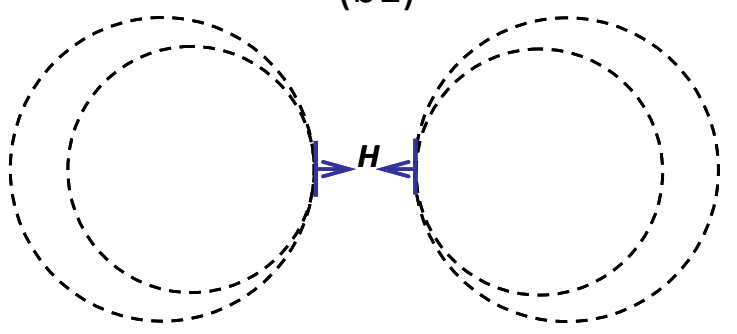

(b2)

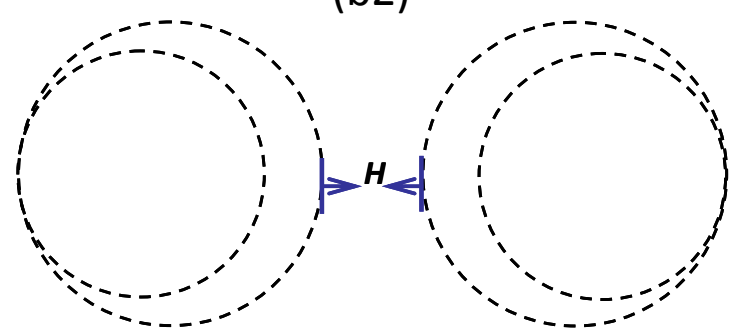

(c1)
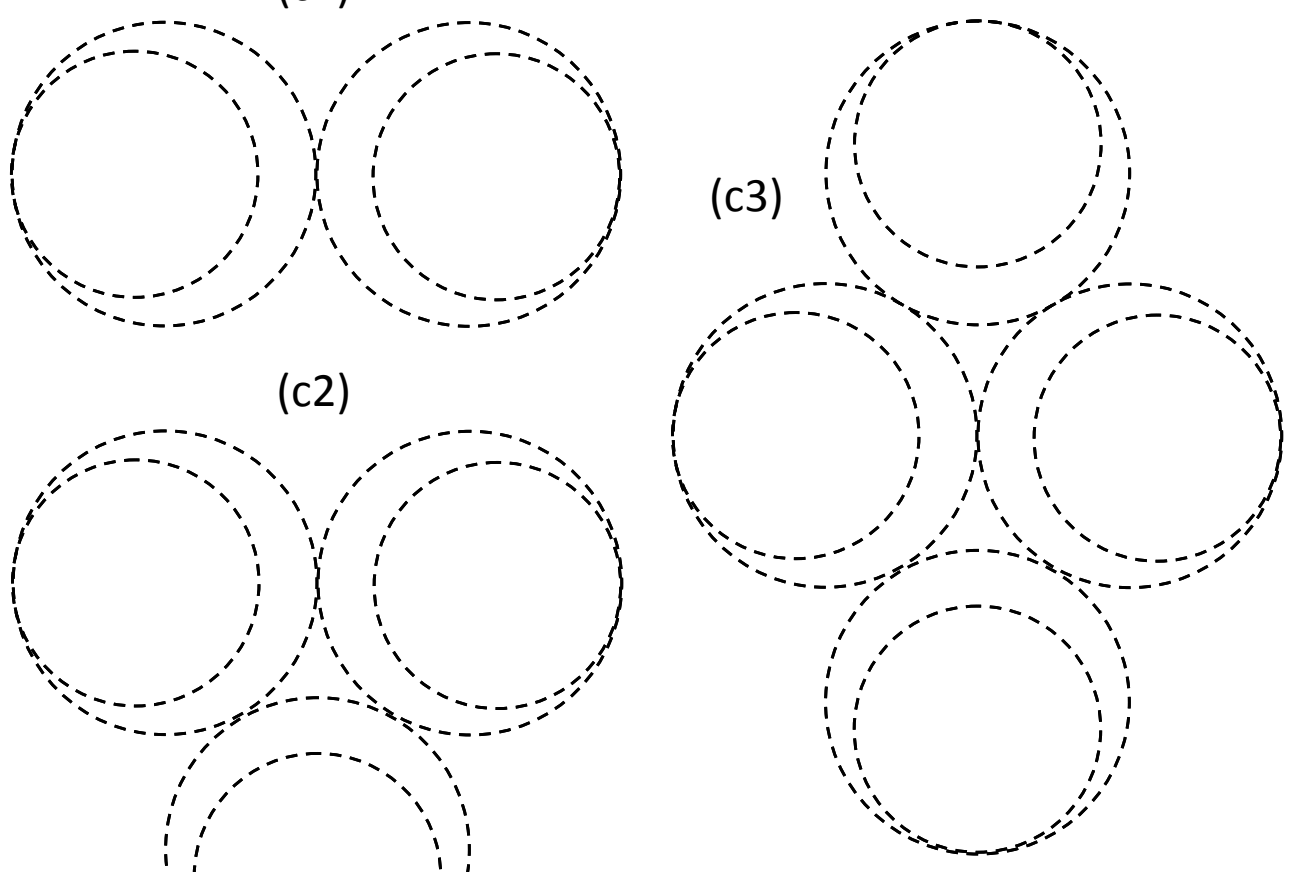

(c2)

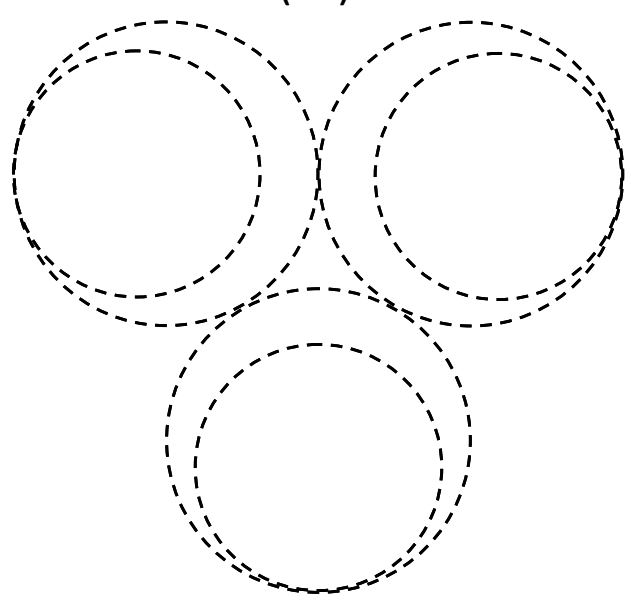

(c3)

Figure S8. (a) Interaction between two CHPs. (b) Interaction between two PHPs. The contact points of the inner and outer spheres of the two interacting PHPs face toward and away from each other in (b1) and (b2), respectively. (c) Aggregated PHPs with the contact points of the inner and outer spheres of each PHP facing outward of the aggregates. 

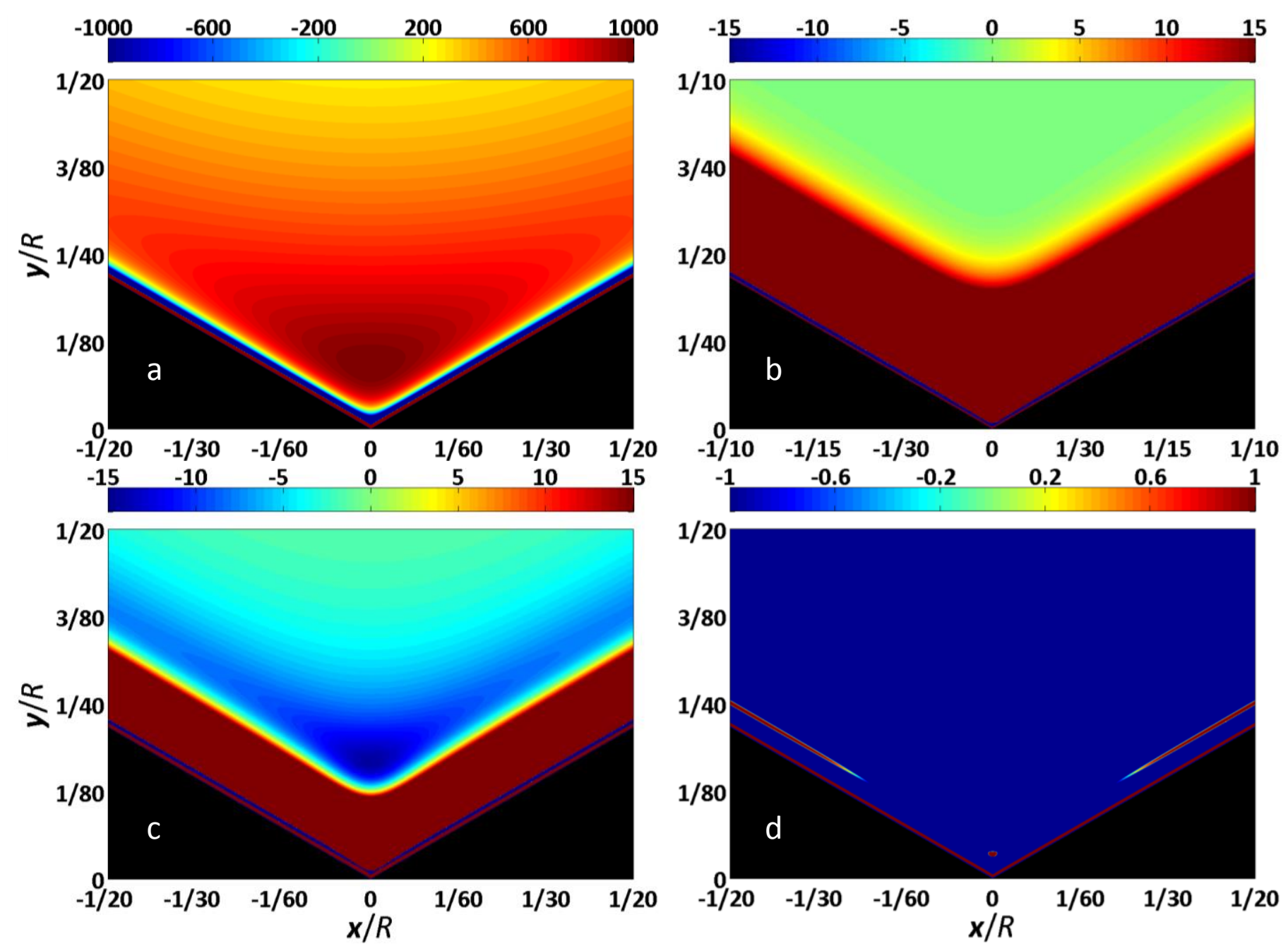

Figure S9. Maps of component of DLVO force along the $y$-direction $\left(F_{\mathrm{y}}\right)$ normalized by a

hydrodynamic drag torque $\left(F_{\mathrm{D}}\right)$ for interactions between a $1000 \mathrm{~nm}$ silica SP and two

intersecting half planes with an angle of 120 degree on the $x-y$ plane at $z=0$ at different

ISs (a, $1 \mathrm{mM}$; b, $10 \mathrm{mM}$; c, $100 \mathrm{mM}$; d, $200 \mathrm{mM}$ ). All simulation conditions are consistent

with those in Figure 5 except the interaction force scales. 

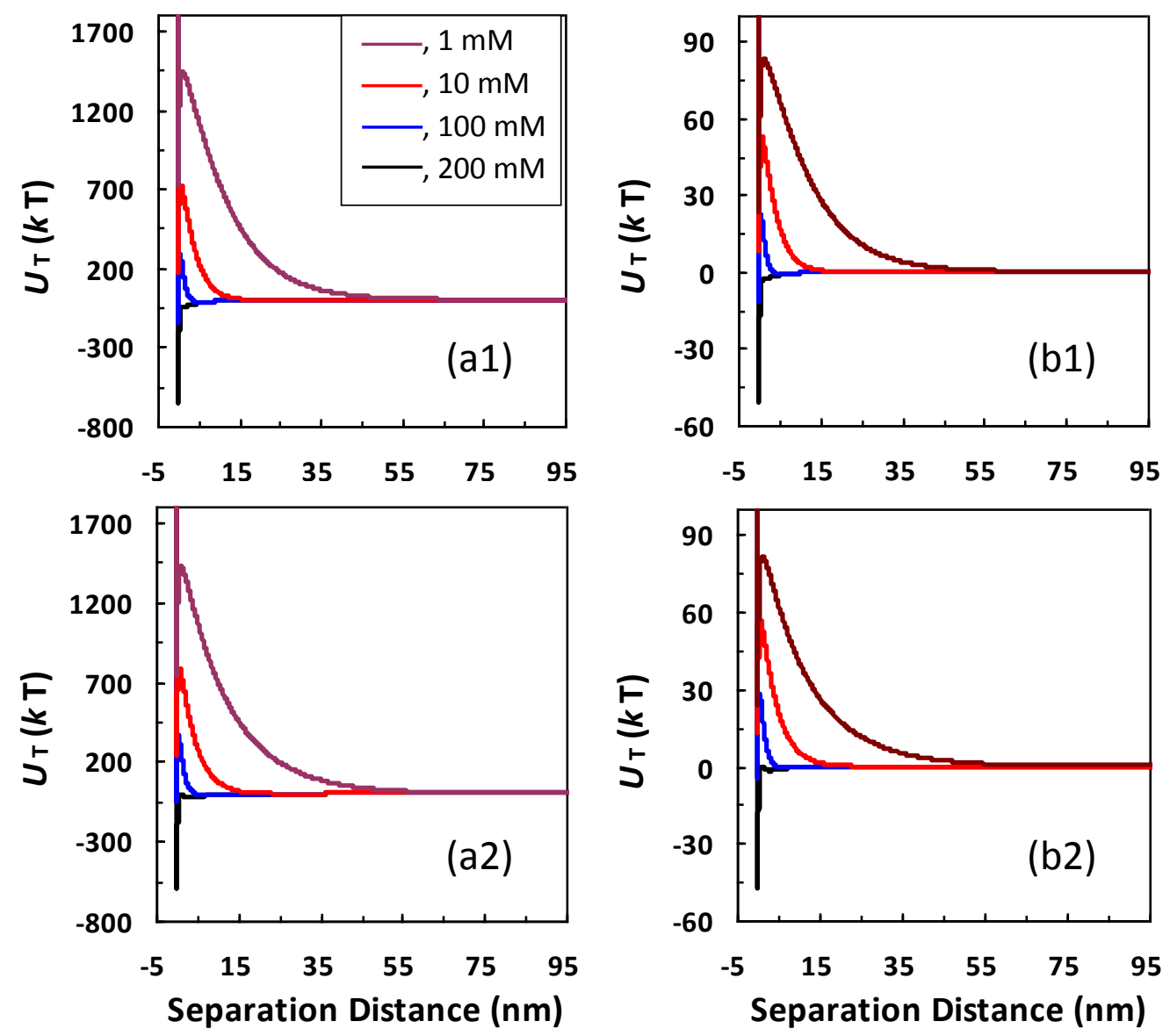

Figure S10. IEPs for the (a) $1000 \mathrm{~nm}$ and (b) $80 \mathrm{~nm}$ SPs at different ISs. The DL interaction energies in (1) were calculated by using Equation (4) or exactly determined by solving the non-linear PB equation using the aforementioned numerical method in (2).

Details about the numerical method can be found in the section of Exact Evaluation of Differential DL Interaction Energies between Two Planar Surfaces in the Supporting Information. 


\section{References}

(1) Bhattacharjee, S.; Elimelech, M.; Borkovec, M. DLVO interaction between colloidal particles: beyond Derjaguin's approximation. Croatica Chimca Acta 1998, 71, 883-903.

(2) Shen, C.; Zhang, M.; Zhang, S.; Wang, Z.; Zhang, H.; Li, B.; Huang, Y. Influence of surface heterogeneities on reversibility of fullerene $\left(\mathrm{nC}_{60}\right)$ nanoparticle attachment in saturated porous media. J. Hazard. Mater. 2015, 290, 60-68.

(3) Devereux, O. F.; de Bruyn, P. L. Interaction of Plane Parallel Double Layers. MIT Press, Cambridge, 1963.

(4) Tiraferri, A.; Sethi, R. Enhanced transport of zerovalent iron nanoparticles in saturated porous media by guar gum. J. Nanopart. Res. 2009, 11, 635-645. 